Research Paper

\title{
Tumor-Infiltrating Immune Cells: Triggers for Tumor Capsule Disruption and Tumor Progression?
}

\author{
Bin Jiang ${ }^{\circledR}$, Jeffrey Mason², Anahid Jewett ${ }^{3}$, Min-ling Liu ${ }^{4}$, Wen Chen ${ }^{4}$, Jun Qiann ${ }^{1}$, Yijiang Ding ${ }^{1}$, Shuqing \\ Ding ${ }^{1}$, Min $\mathrm{Ni}^{1}$, Xichen Zhang ${ }^{5}$, Yan-gao Man ${ }^{5,6} \bowtie$ \\ 1. National Medical Centre of Colorectal Disease, The Third Affiliated Hospital, Nanjing University of Traditional Chinese Medicine \\ (TCM), Nanjing, China. \\ 2. Laboratory of Proteomics and Protein Science, Veterans Affairs Medical Center, Washington, DC, USA. \\ 3. Tumor Immunology Laboratory, Division of Oral Biology and Medicine, Jonsson Comprehensive Cancer Center, UCLA School of Den- \\ tistry, Los Angeles, CA. USA. \\ 4. Pathology and Laboratory Medicine Service, Veterans Affairs Medical Center, Washington, DC, USA. \\ 5. College of Animal Science and Veterinary Medicine, Jilin University, Changchun, Jilin, China. \\ 6. Diagnostic and Translational Research Center, Henry Jackson Foundation, MD, USA.
}

$\triangle$ Corresponding author: Jiang Bin, MD, PhD. Associate Director, National Medical Centre of Colorectal Diseases Associate Professor, University of Traditional Chinese Medicine, The Third Affiliated Hospital, Nanjing University of Traditional Chinese Medicine, Nanjing, China. Phone: 18951755051; E-mail: jbfirsth@yahoo.com.cn; or jbfirsth1@gmail.com Or: Yan-gao Man, MD, PhD. The Diagnostic and Translational Research Center, Henry Jackson Foundation, 401 Professional Drive, Gaithersburg, MD 20879, USA. Phone: 240-833-4969; Fax: 240-833-4940; E-mail: ymann@hjfresearch.org.

(C) Ivyspring International Publisher. This is an open-access article distributed under the terms of the Creative Commons License (http://creativecommons.org/ licenses/by-nc-nd/3.0/). Reproduction is permitted for personal, noncommercial use, provided that the article is in whole, unmodified, and properly cited.

Received: 2013.01.03; Accepted: 2013.02.25; Published: 2013.03.05

\begin{abstract}
Background: Our previous studies of human breast and prostate cancer have shown that aberrant immune cell infiltration is associated with focal tumor capsule disruption and tumor cell budding that facilitate invasion and metastasis. Our current study attempted to determine whether aberrant immune cell infiltration would have similar impact on colorectal cancer (CRC).

Materials and Methods: Tissue sections from 100 patients with primary CRC were assessed for the frequencies of focal basement membrane (BM) disruption, muscularis mucosa (MM) fragmentation, and tumor cell dissemination in epithelial structures adjacent and distal to infiltrating lymphoid aggregates using a panel of biomarkers and quantitative digital imaging.

Results: Our study revealed: (I) epithelial structures adjacent to lymphoid follicles or aggregates had a significantly higher $(p<0.00 \mathrm{I})$ frequency of focally disrupted $\mathrm{BM}$, dissociated epithelial cells in the stroma, disseminated epithelial cells within lymphatic ducts or blood vessels, and fragmented MM than their distal counterparts, (2) a majority of dissociated epithelial cells within the stroma or vascular structures were immediately subjacent to or physically associated with infiltrating immune cells, (3) the junctions of pre-invasive and invasive lesions were almost exclusively located at sites adjacent to lymphoid follicles or aggregates, (4) infiltrating immune cells were preferentially associated with epithelial capsules that show distinct degenerative alterations, and (5) infiltrating immune cells appeared to facilitate tumor stem cell proliferation, budding, and dissemination.

Conclusions: Aberrant immune cell infiltration may have the same destructive impact on the capsule of all epithelium-derived tumors. This, in turn, may selectively favor the proliferation of tumor stem or progenitor cells overlying these focal disruptions. These proliferating epithelial tumor cells subsequently disseminate from the focal disruption leading to tumor invasion and metastasis.
\end{abstract}

Key words: Colorectal cancer; tumor capsule; tumor invasion; metastasis, lymphocyte aggregates. 


\section{Introduction}

It has been well-documented that increased infiltration of immune cells into tumor masses can result in physical destruction of the targeted tumor cells, with a concomitant reduction in tumor burden and improvement in patient prognosis [1-4]. Paradoxical$l y$, it is equally well-documented that pre-invasive epithelial tumors with increased immune cell infiltration have a significantly higher frequency of progression to invasive lesions than their stage- and histopathologically-matched counterparts lacking distinct immune cell infiltrates [5-7]. In addition, a number of in vitro studies have suggested that tumor infiltrating immune cells can promote tumor invasion and metastasis through a number of mechanisms [8-12]. Unfortunately, the direct impact of tumor-infiltrating immune cells and the factors that regulate their ability to either inhibit or promote neoplastic progression remain elusive, making it difficult to judge the clinical significance of tumor-infiltrating immune cells.

As all normal epithelial tissues and in-situ tumors are physically surrounded by a dense continuous capsule that must be breached before physical contact between tumor and immune cells can occur, or before tumor invasion and metastasis can proceed [13-15], we have speculated that the apparent contradiction in the direct impact of tumor infiltrating immune cells may result primarily from the differences in tumor stages and physical locations of infiltrating immune cells. It has been well documented that all the primary infiltrating immune cells, including cytotoxic T-lymphocytes (CTL), natural killer (NK) cells, leukocytes, macrophages, and Mast cells, have to physically contact their targets in order to exert their cytotoxic functions [16-21]. Thus, it is likely that infiltrating immune cells are initially recruited to the tumor site by focal tumor capsule degeneration- and disruption-related alterations, to remove damaged structures. Consistent with our speculation, we found a significant predilection of infiltrating immune cells in tumor nests with focal capsule disruption in breast ( $97 \%$ vs. $22 \%)$ and prostate (91\% vs. $33 \%$ ) than morphologically similar counterparts without disrupted tumor capsules [22-24]. Our subsequent studies revealed that residual breast myoepithelial and prostate basal cells within focally disrupted capsules express a significantly lower level of tumor suppressors and cell proliferation-related proteins, whereas demonstrate a significantly higher rate of degeneration-related changes than their morphologically similar, non-disrupted counterparts [25-32]. Our studies also revealed that the predominant immune cell type associated with epithelial capsules was CTL, which displayed significant cytotoxic impact on degenerative myoepithelial or basal cells, but not on associated luminal cells [25-32]. In contrast, luminal cells overlying focally disrupted capsules have a significantly higher level of proliferation and expression of tumor stem cell- and growth factor-related genes than their adjacent counterparts distant from the disruptions [25-32]. Our more recent study has further shown that denatured collagen I, a main element of tumor capsules, can function as a strong chemoattractant for macrophage recruitment [33].

Together, these findings have led us to propose that aberrant immune cell infiltration at the pre-invasive stage may represent a trigger factor for tumor invasion and metastasis [25-29]. According to our hypothesis, focal degeneration of the epithelial capsule due to age or disease attracts immune cell infiltration, which further degrades the focally degenerating area resulting in a focal disruption within the capsule. If the focal disruption occurs in a region underlying undifferentiated progenitor or tumor stem cells, the changing microenvironment may selectively favor their proliferation, leading to cell "budding" from the disruption and subsequent growth into the stroma or dissemination to vascular structures [25-32]. Our hypothesis has been recognized by a number of internationally recognized experts in the field [34-36] as more compatible with existing experimental evidence than the traditional "protoelytic enzyme" theory [37].

Our current study attempted to expand our observations to colorectal tumors. The hypothesis tested was that as colorectal tissues share very similar structural barriers, the basement membrane (BM) and the muscularis mucosa (MM), and the same hypothesized sequence of tumor progression [38-41], aberrant immune cell infiltration may have the same destructive impact on epithelial capsules and the same promoting impact on tumor progression as those seen in breast and prostate tumors [23-27]. In addition, our study intended to identify direct morphological and immunohistochemical signs suggestive of the potential sub-cellular mechanism(s) of tumor infiltrating immune cells on tumor progression.

\section{Materials and Methods}

De-identified and unstained slides of formalin-fixed, paraffin-embedded (FFPE) consecutive tissue sections were obtained from 100 age- and type-matched CRC patients with and without positive lymph nodes plus five normal controls. All tumor cases were confirmed to be adenocarcinoma and the five controls were confirmed to be free of morphological and immunohistochemical sings of colorectal 
disease. These slides were retrieved under an Institutional Review Board (IRB)-approved protocol (\#2008001) from the Nanjing Municipal Hospital of Nanjing University of Traditional Chinese Medicine (TCM), China. The first and last slides from each tissue block from each case were stained with hematoxylin and eosin (H\&E) for morphological classification using established criteria. The remaining sections were used for immunohistochemical (IHC) assessment. After preliminary examination, 8-cases are excluded due to the lack of sufficient tissue for analysis or tissue detachment from the slides. The clinical parameters of the study population are shown in Table 1.

Table I. Main clinical parameters of patients.

\begin{tabular}{llll}
\hline Parameters & Node positive & Node negative & $\mathbf{p}$ \\
\hline $\begin{array}{l}\text { Mean age (years) } \\
\text { Tumor type }\end{array}$ & 59 & 61 & $>0.05$ \\
$\begin{array}{l}\text { Tumor size (cm) } \\
\begin{array}{l}\text { Depth of invasion } \\
\text { Submucosa and/or }\end{array}\end{array}$ & $4.95 \times 3.68$ & $4.55 X 3.54$ & $>0.05$ \\
$\begin{array}{l}\text { superficial MP } \\
\text { Deep MP and subse- } \\
\text { rosa }\end{array}$ & $33(89.2 \%)$ & $48(87.2 \%)$ & $>0.05$ \\
\hline
\end{tabular}

Double immunohistochemistry was utilized to examine the distribution and interactions among tumor-infiltrating immune cells, tumor capsules, and budding tumor cells. The following antibodies were used in this study: (a) leukocyte common antigen (LCA, clone: 2B11+PD7/26. Dako, Carpinteria, CA), which is expressed by all normal hematopoietic cells and their neoplastic transformations; $(b)$ cytokeratin (CK) AE1/AE3 (clone: AE1/AE3, Dako, Carpinteria, CA), which detects high- and low-molecular weight keratins expressed by all epithelium-derived cells; (C) collagen IV (clone: CIV22, Dako, Carpinteria, CA), which is the main building block of the tumor capsule, (d) D2-40 (clone: D2-40, Signet, Dedham, MA), which is a phenotypic marker for lymphatic endothelium, and (e) CD34 (clone: clone: QBEnd 10; Dako, Carpinteria, CA, USA), which is a phenotypic marker for blood vessel endothelial cells. Double immunohistochemistry was carried out using our previously published methods (42). To assess the specificity of the immunostaining, various negative controls were used, including: (1) substitution of the primary antibody with the same isotype or pre-immune serum of the antibody, and (2) omission of the secondary antibody. All immunostaining procedures were repeated 2-3 times under identical conditions.
Lymphoid follicles, lymphocyte aggregates, and tumor-infiltrating lymphocytes were defined according to commonly accepted criteria [38]. A focal disruption in the $\mathrm{BM}$ or a fragmentation in the MM was defined as a physical gap in these structures, larger than the combined diameter of at least three epithelial cells in at least three consecutive tissue sections. Dissociated tumor cells were defined as isolated individual cells, or small clusters of cells (few than15 cells/cluster) observed to be physically separated from the tumor core in at least three consecutive tissue sections.

To compare the frequencies of focal BM disruptions, MM fragmentations, and dissociated tumor cells in structures adjacent to and distant from, infiltrating lymphoid follicles and large immune cell aggregates, the entire epithelium, lamina propria (LP), and $\mathrm{MM}$ of the pre-invasive tissue component was digitally photographed. The digital images were enlarged to 400X and reviewed under the screen of a standard computer. The mean frequencies of focal BM disruptions, MM fragmentations, and dissociated tumor cells between these tissue sites were statistically compared with the Pearson's Chi-square test. Statistical significance was defined as $p<0.05$.

To assess the correlation of immune cell infiltration with tumor invasion, all junctions of pre-invasive and invasive lesions were identified and examined to determine whether these junctions are exclusively or preferentially associated with infiltrating immune cell aggregates.

To identify the direct morphological and immunohistochemical signs suggestive of the potential sub-cellular mechanism(s) of infiltrating immune cells on tumor progression, tissue sections from 10 selected cases were assessed with three panels of bio-markers. First, they were subjected to triple immunohistochemistry with vascular structure-related markers, including D2-40, CD34, and CD31 (clone: JC70A). Immunostained sections were examined to determine whether increased immune cell infiltration is associated with an increased vascular density, which has been suggested to promote tumor invasion and metastasis. Second, they were subjected to triple immunohistochemistry with an epithelial capsule marker, collagen IV and markers to immune cells (CD8, clone: C8/144B; CD16, clone: DJ130C; Mast cell tryptase, clone: AA1. Dako, Carpinteria, CA, USA). Immunostained sections were examined to determine whether these immune cells are physically associated with the epithelial capsules and whether associated capsules show degenerative alterations. Third, they were subjected to triple immunohistochemistry with phenotypic markers to immune cells, stem cell 
(CD133, clone: AC133, Miltenyi Biotec, Auburn, CA, USA; CK-19, clone: RCK108, Dako, Carpinteria, CA, USA), and proliferation marker Ki-67 (clone: MIB1) (Dako, Carpinteria, CA, USA). Immunostained sections were examined to determine whether increased immune cell infiltration is associated with increased epithelial cell proliferation, budding, and dissemination.

\section{Results}

In sections immunostained for collagen IV, the $\mathrm{BM}$ and $\mathrm{MM}$ in a vast majority of the normal and pre-invasive epithelial structures distant from lymphoid follicles or infiltrating immune cell aggregates were presented as a dense and continuous band, which completely encircles the colonic crypts and segregates the mucosae from the submucosae (Figure 1A-B). In sections double immunostained for infiltrating immune cells and epithelial capsules, immune cell infiltration and focal disruptions of the BM were readily appreciable in some normal colonic epithelial structures. However, most infiltrates distant from the tumor tissue were located at the middle or upper portion of the mucosae, and the BM in epithelial structures near the MM was generally intact (Figure 1C-D). In addition, focal BM disruptions distant from the tumor tissue were facing the luminal direction, and the MMs were largely intact with no distinct immune cell infiltration (Figure 1E-F). In contrast, focal BM disruptions adjacent to the tumor tissue were generally facing the basolateral direction, and the MMs were often focally fragmented or separately with extensive immune cell infiltration within the MMs (Figure 1G-H).

The frequency and extent of focal BM and MM attenuation or disruptions appeared to increase with increasing extent of immune cell infiltration and increasing degree of malignancy. As shown in Figure 2, both the BM and $\mathrm{MM}$ in normal appearing epithelial structures distant from the invasive CRC are largely intact with fewer infiltrating immune cells. In contrast, both the BM and MM in normal appearing epithelial structures immediately adjacent to the invasive CRC are focally attenuated or disrupted with extensive immune cell infiltration. These infiltrating immune cell aggregates harbor several epithelial cell clusters with no distinct surrounding BM (Figure 2B).

A vast majority of the MM distant from lymphoid follicles was well defined and continuous. In sharp contrast, nearly all MMs immediately adjacent to lymphoid follicles exhibited aberrant alterations. As shown in Figure 3, all the MMs immediately adjacent to 7-lymphoid follicles in two cases are either totally absent or focally disrupted and variable num- bers of dissociated individual epithelial cells or cell clusters are seen within or immediately adjacent to these lymphoid follicles.

Nearly all epithelial structures distant from focally disrupted MMs were uniform in size with a distinct lumen, and were surrounded by a continuous BM. In sharp contrast, variable numbers of solid epithelial cell clusters or dissociated individual cells lacking BM were seen overlying focally disrupted MMs, and some of these cells or cell clusters appeared to have migrated into the center of the lymphoid follicles through the focal MM disruption (Figure 4).

Within or immediately adjacent to lymphoid follicles, the vascular density was significantly increased, compared to that seen in tissues distant from these follicles. As shown in Figure 5, these lymphoid follicles not only harbor a variable number of dissociated epithelial cells, but also harbor large open lumen vascular structures with disseminated cells. Over 75\% of dissociated epithelial cells were either immediately adjacent to or physically associated with infiltrating Immune cells.

Those dissociated epithelial cell clusters within the lymphocyte follicles or aggregates overlying focally disrupted MM (as shown in Figures 3-5) appeared to be at increased risk to progress to invasive CRC. As shown in Figure 6, invasive CRC is exclusively seen near the normal appearing epithelial structures physically associated with infiltrating immune cell aggregates. The infiltrating immune cell aggregate in each of two cases harbor multiple epithelial cell clusters that have no distinct surrounding $\mathrm{BM}$, and they also share similar morphological features with the adjacent invasive CRC.

The risk of progression to invasive CRC for those dissociated epithelial cells or cell clusters was further suggested by the fact that all 35 morphologically distinct pre-invasive and invasive junctions detected in 24 cases were located within or immediately adjacent to lymphoid follicles or infiltrating immune cell aggregates. As shown in Figures 7-9, most of these dissociated cells or invasive cancer cells are either immediately adjacent to or physically associated with infiltrating immune cells, and dissociated epithelial cells in the mucosa migrate into the submucosa through focally disrupted MMs. No similar dissociated epithelial cells or pre-invasive and invasive junctions were seen in tissues that completely lacked lymphoid follicles or large infiltrating immune cell aggregates.

Increased immune cell infiltration appeared to stimulate lymphangiogenesis and angiogenesis, which facilitate intravasation of dissociated epithelial 
cells. As shown in Figure 10, in morphologically comparable tissues, both the density and luminal size of lymphatic ducts are substantially higher and larger in tissues adjacent to than those distant from infiltrating immune cell aggregates. Similar findings were obtained with phenotypic markers CD31 and CD34 for blood vessel endothelium (data not shown).

Infiltrating CTL, NK, and Mast cells were preferentially seen within the normal appearing or pre-invasive tissue component and a majority of infiltrating immune cells were physically associated with the epithelial capsules, which often show distinct degenerative alterations. As shown in Figure 11, most infiltrating NK and Mast cells are located at or near the site of focally disrupted epithelial capsules, which harbor several dissociated epithelial cell clusters that lack distinct surrounding BM. In contrast, the BM distant from infiltrating NK and Mast cells is morphologically distinct and continuous.

Increased immune cell infiltration also appeared to be associated with increased epithelial cell proliferation. As shown in Figure 12, a majority of proliferating cells in normal appearing tissues are either physically associated with or immediately adjacent to infiltrating immune cells. In addition, these tissues harbor large open lumen lymphatic ducts with proliferating disseminated cells (Figure $12 \mathrm{~A}-\mathrm{B}$ ).

Increased immune cell infiltration appeared to facilitate tumor stem cell budding and dissemination. In sections double immunostained with markers of stem cells, blood vessel endothelial cells, or infiltrating immune cells, some isolated CK-19-positive cell clusters were seen within an overall CK-19-negative background. These CK-19-positive cell clusters within normal appearing epithelial structures are morphologically and immunohistochemically similar to adjacent invasive cancer cells and also to disseminated cells within the vascular structure (Figure 13). Nearly all these dissociated CK-19-positive cell clusters were either surrounded by or were physically associated with infiltrating immune cells and were also in direct physical continuity with invasive cancers within the submucosa (Figure 14). The results of CD 133 immunostaining were inconsistent (data not shown).

A total of 91 lymphoid follicles and large lymphocyte aggregates were seen in 33 of 92 cases. All laminar propria (LP) immediately adjacent to these lymphoid follicles and lymphocyte aggregates harbored normal epithelial structures with focally attenuated or disrupted BM, or dissociated cells or cell clusters. In contrast, only 9 (10\%) LPs distant from these follicles and aggregates harbored similar structures (Table 2; $p<0.001$ ). Similarly, all MM immediately adjacent to these follicles and aggregates were focally attenuated or fragmented, while only $9(10 \%)$ MM distant from these lymphoid follicles showed similar alterations (Table 3, $p<0.001$ ). The morphology and distribution of infiltrating lymphocytes on the $\mathrm{BM}$ and $\mathrm{MM}$ of both node positive and negative cases appeared to be similar, except that lymphatic ducts with disseminated cells were exclusively seen in the lamina propria of node positive cases and a single 'node negative' case with confirmed CRC metastasis.

Table 2. Focal BM disruptions adjacent to and distant from lymphoid follicles and aggregates.

\begin{tabular}{llll}
\hline Location & $\begin{array}{l}\text { Lymphoid follicles \& } \\
\text { aggregates }\end{array}$ & $\begin{array}{l}\text { Epithelia with focal BM } \\
\text { disruptions }\end{array}$ & $p$ \\
\hline $\begin{array}{l}\text { Adjacent } \\
\text { to }\end{array}$ & 91 & $91(100 \%)$ & $<$ \\
$\begin{array}{l}\text { Distant } \\
\text { from }\end{array}$ & 91 & $9(10 \%)$ & 0.001 \\
\hline
\end{tabular}

Table 3. Focal MM disruptions adjacent to and distant from lymphoid follicles and aggregates.

\begin{tabular}{llll}
\hline Location & $\begin{array}{l}\text { Lymphoid follicles \& } \\
\text { aggregates }\end{array}$ & $\begin{array}{l}\text { Focal MM attenuation \& } \\
\text { disruptions }\end{array}$ & $p$ \\
\hline $\begin{array}{l}\text { Adjacent } \\
\text { to }\end{array}$ & 91 & $91(100 \%)$ & $<$ \\
$\begin{array}{l}\text { Distant } \\
\text { from }\end{array}$ & 91 & $9(10 \%)$ & 0.001 \\
\hline
\end{tabular}

\section{Discussion}

Consistent with our hypothesis, our current study has detected almost identical frequency and pattern of immune cell infiltration and associated epithelial capsule disruptions as those seen in our previous studies of human breast and prostate tumors [23-32]. In addition, our current study has yielded the following unique findings: (1) normal appearing epithelial structures immediately adjacent to lymphoid follicles and infiltrating immune cell aggregates have a significantly higher frequency of focally attenuated or disrupted BM, dissociated cells within lymphatic ducts or blood vessels, and focally fragmented MM than their distant counterparts, (2) a vast majority of dissociated epithelial cells within the stroma or vascular structures are either immediately adjacent to or physically associated with infiltrating immune cells, $(\underline{3})$ the physical conjunctions of pre-invasive and invasive lesions are exclusively seen at the sites of lymphoid follicles and infiltrating immune cell aggregates, $(\underline{4})$ aberrant immune cell infiltration is predominantly seen in normal appearing epithelial structures with focally disrupted BM and MM, (ㅁ) 
infiltrating CTL, NK, and Mast cells are preferentially associated with the epithelial capsules, which is often associated with distinct degenerative alterations, (6) infiltrating immune cells appear to substantially stimulate lymphangiogenesis and angiogenesis, and (7) increased immune cell infiltration is associated with elevated stem cell proliferation, budding, and dissemination. Together, these findings suggest that tumor infiltrating immune cells may have promoting impact on tumor invasion and metastais.

The promoting impact of tumor infiltrating immune cells on tumor progression seen in our current study is in agreement with previous clinical observations that pre-invasive epithelial tumors with increased immune cell infiltration have a significantly higher frequency of subsequent invasive lesions than their stage- and histopathologically-matched counterparts without distinct immune cell infiltration [5-7]. The findings of the mechanistic assays in our current study are also consistent with those of others, which have shown that aberrant immune cell infiltration could promote tumor invasion and metastasis through a number of mechanisms: (a) macrophage-enhanced cancer cell migration through secretion of chemotactic and chemokinetic factors known to promote angiogenesis and fibrillogenesis, allowing tumor cells to track along collagen fibers to blood vessels $[8,9],(\underline{b})$ macrophage digestion of tumor cells, resulting in the mixture of genetic material that creates a hybrid phenotype, which can metastasize to remote sites [10], and (c) infiltrating immune cell release of growth factors and other proliferation-related molecules to associated tumor cells through direct physical contact $[11,12]$. To the best of our knowledge, however, our study is the first one to suggest that the primary impact of tumor-infiltrating immune cells may be associated with the physical destruction of the tumor capsule. As the disruption of the tumor capsule is an absolute pre-requisite for invasion or metastasis of all epithelium-derived tumors, our findings appear to have unique scientific and clinical implications, compared to previously published findings and theories regarding the impact of tumor-infiltrating immune cells.

The BM is composed of type IV collagen, laminins, and other molecules that form a continuous fibrous capsule, which attaches to the normal epithelial cells and their derived pre-invasive tumor cells, physically segregating these cells from the surrounding stroma [13-17]. As the epithelial component is normally devoid of blood vessels and lymphatic ducts, the disruption of the BM is a pre-requisite for tumor invasion or metastasis [13-17]. The colorectal MM consists of two morphologically distinct smooth muscle layers that form a dense band-like structure, which physically separates the mucosa from the submucosa [38, 43-46]. Although its structural features have been well described, the physiological functions of the MM remain elusive [38, 43, 44]. However, it has been suggested that the MM might have an important inhibitory role on tumor progression [43-46]. In human CRC, a focal disruption or absence of the $\mathrm{MM}$ is a pre-requisite for migration of invasion-initiating cells from the mucosa into the submucosa [38, 43-46].

Because of these structural features, a focal disruption in the BM and MM could potentially have several consequences: (a) altered permeability for oxygen and nutrients [47], (b) direct epithelial-stromal cell contact [48], (c) direct exposure of epithelial cells to different growth factors [49], and ( $\underline{\mathrm{d}})$ an open path allowing the migration of epithelial cells from the mucosa into the submucosa. As the adult gut stem cell population is basally located near the MM [50-55], these alterations may individually or collectively elicit the exit of stem cells from quiescence leading to increased proliferation, which could have two fundamentally different consequences. On one hand, it may represent the normal colonic replenishment process, as the BM towards the luminal direction has to be degraded or disrupted in order to allow the cells at the base of the crypt growing upwards to replace aged or damaged apical luminal cells. On the other hand, it may selectively favor invasion and dissemination of the overlying tumor stem cells or a biologically more aggressive cell clone. The fate of the consequences of a given BM disruption is likely to be determined not only by the genetic profile of the overlying epithelial cells, but also by the physical location of the epithelial structure. The epithelial structures shown in Figure 1E-F are likely to represent a normal replenishment process as the focal BM disruptions are facing the luminal direction and the MM is densely packed and continuous. In contrast, the structures shown in Figures $1 \mathrm{G}-\mathrm{H}, 2 \mathrm{~B}, 6-8,11$, and 12 are likely to be at increased risk for invasion as (1) the focal BM disruptions are facing the basolateral direction, (2) they harbor budding or dissociated cells, and (3) the MM is loosely packed with "channel"-like spaces containing a significant number of filtrating immune cells.

Although these alterations are only microscopic, they may signify aberrant expression of the building-blocks of the BM and MM. It has been well documented that the expression levels of both mRNAs and proteins of the BM subunits changes substantially during colorectal carcinogenesis and are significantly down-regulated during CRC invasion [56-58]. It has also been reported that the expression of the collagen 
IV or collagen I mRNAs or proteins can directly and significantly impact the lineage differentiation of both adult and embryonic stem cells [59-62]. A previous study assessed the physical integrity of the BM with immunohistochemistry for collagen IV and laminin (two primary components of the BM) and attempted to correlate BM disruptions with the survival of patients with different stage CRC [63]. The study revealed that a total loss or considerable discontinuity of the BM was adversely correlated with survival, whereas no corresponding association of outcome with the stage of disease was evident [63].

The mechanism(s) and specific molecule(s) for the recruitment of tumor-infiltrating immune cells to the site of epithelial structures with focally degenerated BM and MM cannot be determined by our current study, while it is most likely that immune cell infiltration is triggered by the convergence of tissue injury, innate immune response to that injury, and the presence of tumor stem cells at the site of the injury. Our previous studies have shown that a denatured component of the BM, collagen I, could act as a strong chemoattractant for the recruitment of macrophages, suggesting that degenerated tumor capsule elements may function as chemo-attractants for tumor-infiltrating immune cells [33]. After focal disruption of the tumor capsule by infiltrating immune cells and the exposure of the overlying epithelial cells to the adjacent stroma, additional immune cells may be recruited to the site resulting from the accumulation of microvesicles on the surface of the tumor cell plasma membrane [64]. Microvesicles released from tumor cell membranes may contain proteins that function as self-epitopes activating a subset of immune cells. Prior to their release, these microvesicles are embedded within the tumor cell membrane, and could potentially function as receptors to attract immune cell infiltration.

It was interesting to note that although distinct signs of degeneration were seen in epithelial capsules physically associated with infiltrating immune cells, whereas no distinct degenerative alterations were observed in epithelial cells physically associated with or surrounded by infiltrating immune cells. The underlying mechanism(s) for the differential impact of immune cells on epithelial capsules and epithelial cells is unknown, while it could potentially result from two main factors: (a) the lack of tumor antigenic proteins on the surface of the associated epithelial cells. As those cells are located exclusively at or near focally disrupted epithelial capsules, they may represent a population of tumor progenitors or stem cells that are not "mature" enough to express a measurable amount of surface proteins on their plasma member
$[25,26]$. Consistent with our speculation is the fact that our previous gene expression profiling with freshly frozen prostate and breast tissues have shown that tumor cells overlying focally disrupted capsules have a 5.03- and 5.45-fold increase in the expression level of two stem cell lineage markers, KIT and NCOR2, respectively $[25,26]$, and (b) the presence of a subtype(s) of immune cells that lack cytotoxic functions. Immune cells physically associated with epithelial cells are likely to represent a population of $\mathrm{CD} 4(+) /$ CD25(+)/Foxp3(+) regulatory $\mathrm{T}$ cells or CD8(+)/CD28(-) cells. Recent studies have shown that the number of these infiltrating immune cells increases with tumor progression, and that these cells can induce tolerance to tumor cells through cell-to-cell interactions or by secreting different cytokines, which suppress the cytotoxic functions of the immune cells [65].

Focal BM disruptions and MM fragmentations seen in our current study in normal appearing colorectal tissues are consistent with those of our previous studies, which have shown that $15-30 \%$ of patients with or without malignant prostate or breast lesions harbor morphologically normal epithelial structures that show extensive focal capsule disruptions and budding cells [22-25]. The intrinsic entity of these structures is unknown, but they are most likely to represent the previously undefined malignant phenotype, or pending malignant lesions with pre-disposition of genetic abnormalities. Our previous study revealed that breast clinging ductal carcinoma in situ (DCIS) share a similar molecular and clinical profile with other types of DCIS, while it is often morphologically indistinguishable from normal breast epithelial structures [66]. Previous studies from others have also shown that: (1) the prostate tissues in a subset of aged men or normal-appearing prostate tissues adjacent to prostate cancer harbor a DNA phenotype that is identical to invasive and metastatic prostate cancer [67], and (2) cancer of unknown primary site (CUP) is one of the 10 most frequent cancers and is ranked as the $4^{\text {th }}$ commonest cause of cancer deaths, but its primary tumor site remains elusive [68]. It is interesting to note that the dominant histological type of both CUP and CRC are adenocarcinoma $[69,70]$, raising a possibility that some of CUP may originate from CRC.

In summary, our current study suggest that at the early stage of colorectal carcinogenesis, tumor infiltrating immune cells may be recruited to the tumor site by the degenerated products of the tumor capsule due to age or disease. Infiltrating immune cells degrades the degenerating capsule resulting in the formation of a focal disruption in the capsule, 
which selectively favors proliferation, "budding", and dissemination of the underlying stem cells. Consequently, aberrant immune cell infiltration into the pre-invasive tumors is likely to directly and significantly promote tumor invasion into the stroma. Our findings could reasonable explain the contradictory reports and statements regarding the impact and clinical significance of immune cell infiltration into tumor tissues. More importantly, as the disruption of the tumor capsule is an absolute prerequisite for tumor invasion and metastasis, local or systematic administration of anti-inflammatory agents to prevent immune cell infiltration-induced capsule destruction may be beneficial in preventing tumor progression. A recent report published in Lancet Oncology has revealed that regular use of Aspirin, a non-steroidal anti-inflammatory drug, reduces the long-term risk of $\mathrm{CRC}$ and other cancer and the risk of distant metastasis [71].

\section{Acknowledgements}

This study was supported in part by grants 200601060, 201108004 from Science and Technology Bureau of Nanjing, ZKX11004from Health Bureau of Nanjing, LZ11105 from Jiangsu Province Bureau of Traditional Chinese Medicine to Jiang Bin. This study was also supported in part by grants 2008-02 from the US Military Cancer Institute and Henry M. Jackson Foundation and 2006CB910505 from the Chinese Ministry of Science and Technology to Yan-gao Man and Xichen Zhang, by Merit Award ONCA-037-10F from the Veterans Health Administration awarded to Jeff Mason, and by RO1-DE12880 from NIDCR-NIH to Anahid Jewett.

\section{Competing Interests}

The authors have declared that no competing interest exists.

\section{References}

1. Topalian SL, Solomon D, Rosenberg SA. Tumor-specific cytolysis by lymphocytes infiltrating human melanomas. I Immunol. 1989; 142(10):3714-25.

2. Baxevanis CN, Dedoussis GV. Papadopoulos NG, Missitzis I,Stathopoulos GP, Papamichail M. Tumor specific cytolysis by tumor infiltrating lymphocytes in breast cancer. Cancer. 1994; 74:1275-82.

3. Chen LJ, Zheng X, Shen YP et al. Higher numbers of T-bet(+) intratumoral lymphoid cells correlate with better survival in gastric cancer. Cancer Immunol Immunother. 2012; [Epub ahead of print].

4. Milne K, Alexander C, Webb JR et al. Absolute lymphocyte count is associated with survival in ovarian cancer independent of tumor-infiltrating lymphocytes. J Transl Med. 2012 Feb 27;10:33.

5. Gannot G, Gannot I, Vered H, Buchner A, Keisaris Y. Increase in immune cell infiltration with progression of oral epithelium from hyperkeratiosis to dysplasia and carcinoma. Br J Cancer. 2002; 86: 1444-8.

6. MacLennan GT, Eisenberg R, Fleshman RL et al. The influence of chronic inflammation in prostatic carcinogenesis: a 5-year follow-up study. J Urol. 2006; 176: 1012-6.

7. Smith CJ, Gardner WA. Jr. Inflammation-proliferation: possible relationships in the prostate. Prog Clin Biol Res. 1987; 239: 317-25.
8. Pollard JW. Tumour-educated macrophages promote tumour progression and metastasis. Nat Rev Cancer. 2004; 4: 71-8.

9. Wyckoff J, Wang W, Lin EY, et al. A paracrine loop between tumor cells and macrophages is required for tumor cell migration in mammary tumors. Cancer Res. 2004; 64: 7022-9.

10. Pawelek JM, Chakraborty AK. The cancer cell--leukocyte fusion theory of metastasis. Adv Cancer Res. 2008; 101: 397-444.

11. De Narddo DG, Johansson M, Coussens LM. Immune cells as mediators of solid tumor metastasis. Cancer Metastasis Rev. 2008; 27: 11-8.

12. Eiró N, Vizoso FJ. Inflammation and cancer. World J Gastrointest Surg. 2012; 4(3):62-2.

13. Lazaris ACh, Tzoumani AN, Thimara I et al. Immunohistochemical assessment of basement membrane components in colorectal cancer: prognostic implications. J Exp Clin Cancer Res.2003; 22(4): 599-606.

14. Ambiru S, Miyazaki M, Ito H, et al. Type IV collagenase activities in human colorectal cancers and its role in cancer invasion and metastasis. Nihon Shokakibyo Gakkai Zasshi. 1993;90(7): 1555-61.

15. Hagedorn EJ, Sherwood DR. Cell invasion through basement membrane: the anchor cell breaches the barrier. Curr Opin Cell Biol. 2011; 23(5):589-96.

16. Mayadas TN, Tsokos GC, Tsuboi N. Mechanisms of immune complex-mediated neutrophil recruitment and tissue injury. Circulation. 2009; 120(20):2012-24.

17. Waterhouse NJ, Clarke CJ, Sedelies KA, Teng MW, Trapani JA. Cytotoxic lymphocytes; instigators of dramatic target cell death. Biochem Pharmacol.2004; 68(6):1033-40.

18. Cullen SP, Brunet M, Martin SJ. Granzymes in cancer and immunity. Cell Death Differ.2010; 17(4):616-23.

19. Topham NJ, Hewitt EW. Natural killer cell cytotoxicity: how do they pull the trigger? Immunology.2009; 128(1):7-15.

20. de Saint Basile G, Ménasché G, Fischer A. Molecular mechanisms of biogenesis and exocytosis of cytotoxic granules. Nat Rev Immunol.2010; 10(8):568-79.

21. Smyth MJ, Cretney E, Kelly JM et al. Activation of NK cell cytotoxicity. Mol Immunol. 2005; 42(4):501-10.

22. Man YG, Sang QX. The significance of focal myoepithelial cell layer disruptions in human breast tumor invasion: a paradigm shift from the 'proteasecentered' hypothesis. Exp Cell Res. 2004; 301: 103-18.

23. Yousefi M, Mattu R, Gao C, Man YG. Mammary ducts with and without focal myoepithelial cell layer disruptions show a different frequency of white blood cell infiltration and growth pattern: Implications for tumor progression and invasion. AIMM. 2005; 13:30-7.

24. Man YG, Shen T, Zhao YG, Sang QX. Focal prostate basal cell layer disruptions and leukocyte infiltration are correlated events: A potential mechanism for basal cell layer disruptions and tumor invasion. Cancer Detect Prev. 2005; 29: 161-9.

25. Man YG. Focal degeneration of aged or injured myoepithelial cells and the resultant auto-immunoreactions are trigger factors for breast tumor invasion. Med Hypoth. 2007; 69: 1340-57.

26. Man YG, Gardner WA. Focal degeneration of basal cells and the resultant auto-immunoreactions: a novel mechanism for prostate tumor progression and invasion. Med Hypoth. 2008; 70: 387-408.

27. Man YG, Harley R, Mason J, Gardner WA. Contributions of leukocytes to tumor invasion and metastasis: the "piggy-back" hypothesis. Cancer Epidem. 2010; 34: 3-6.

28. Man YG, Mason J, Harley R et al. Leukocyte-facilitated tumor dissemination: a novel model for tumor cell dissociation and metastasis. J Cell Biochem.2011; 112: 1154-67.

29. Man YG. Aberrant leukocyte infiltration: a direct trigger for breast tumor invasion and metastasis. Int J Biol Sci. 2010; 6(2):129-32.

30. Hsiao YH, Deng CX, Mason JT, Chou MC, Man YG. Hidden malignant cells within leukocyte aggregates in normal or hyperplastic tissues of pregnancy-associated breast cancer. Cancer Epidemiol. 2011; [Epub ahead of print].

31. Song G, Ren I, Stojadinovic A et al. Conjunction of tumor cells with lymphocytes: implications for tumor invasion and metastasis. Cancer Epidemiol. 2012; 36(4):354-63.

32. Man YG. Tumor cell budding from focally disrupted tumor capsule: a common pathway for all breast cancer subtype derived invasion? J Cancer. 2010; 1:27-31

33. O'Brien JO, Lyons $\mathrm{T}$, Bell KP et al. Alternatively activated macrophages and collagen I remodeling characterize the post-partum involuting mammary gland across species. Am J Pathol. 2010; 176: 1241-55.

34. Schedion P. Pregnancy-associated breast cancer and metastasis. Nat Rev Cancer. 2006; 6(4):281-91.

35. Min $\mathrm{Hu}$ and Kornelia Polyak. Microenvironmental regulation of cancer development. Curr Opin Genet Dev. 2008; 18(1): 27-34. 
36. Puspa Raj Pandey PR, Saidou J, Watabe K. Role of myoepithelial cells in breast tumor progression. Front Biosci. 2011; 15: 226-36.

37. Goldfarb RH, Liotta LA. Proteolytic enzymes in cancer invasion and metastasis. Semin Thromb Hemost. 1986; 12(4):294-307

38. Noffsinger A, Fenoglio-Preiser CM, Maru D, Gilinsky N. Gastrointestinal Diseases. Washington DC: American Registry of Pathology and Armed Forces Institute of Pathology. 2007.

39. Michor F, Iwasa Y, Rajagopalan H, Lengauer C, Nowak MA. Linear model of colon cancer initiation. Cell Cycle. 2004; 3(3):358-62.

40. Aaltonen LA. The multistep process of colon carcinogenesis. Cytokines Mol Ther.1996; 2(2): 111-4.

41. Luebeck EG, Moolgavkar SH. Multistage carcinogenesis and the incidence of colorectal cancer. Proc Natl Acad Sci USA. 2002; 99(23):15095-100.

42. Man YG, Burgar A. An antigen unmasking protocol that satisfies both immunohisto-chemical and subsequent molecular biological assessments. Pathol Res Pract 2003; 199: 815-25.

43. Uchida K, Kamikawa Y. Muscularis mucosae - the forgotten sibling. J Smooth Muscle Res. 2007; 43(5):157-77.

44. Nagai K, Noguchi T, Hashimoto T, Uchida $Y$, Shimada T. The organization of the lamina muscularis mucosae in the human esophagus. Arch Histol Cytol. 2003; 66(3):281-8.

45. Yoshinaka H, Shimazu H, Fukumoto T, Baba M. Superficial esophageal carcinoma: a clinicopathological review of 59 cases. Am J Gastroenterol. 1991; 86(10):1413-8.

46. Araki K, Ohno S, Egashira A et al. Pathologic features of superficial esophageal squamous cell carcinoma with lymph node and distal metastasis. Cancer. 2002; 94(2):570-5.

47. Csete M, Walikonis J, Slawny N. Oxygen-mediated regulation of skeletal muscle satellite cell proliferation and adipogenesis in culture. J Cell Physical. 2001; 189: 189-96.

48. Sato T, Sakai T, Noguchi Y, Takita M, Hirakawa S, Ito A. Tumor-stromal cell contact promotes invasion of human uterine cervical carcinoma cells by augmenting the expression and activation of stromal matrix metalloproteinases. Gynecol Oncol.2004; 92: 47-56

49. Klos KS, Wyszomierski SL, Sun M et al. c-erbB2 increases vascular endothelial growth factor protein synthesis via activation of mammalian target of rapamycin/p70S6K leading to increased angiogenesis and spontaneous metastasis of human breast cancer cells. Cancer Res. 2006; 6: 2028-2037

50. Yen TH, Wright NA. The gastrointestinal tract stem cell niche. Stem Cell Rev.2005; 2(3):203-12

51. Fevr T, Robine S, Louvard D, Huelsken J. Wnt/beta-catenin is essential for intestinal homeostasis and maintenance of intestinal stem cells. Mol Cell Biol. 2007; 27(21):7551-9.

52. Freeman HJ. Crypt region localization of intestinal stem cells in adults. World J Gastroenterol.2008; 14(47):7160-2.

53. Dhawan P, Ahmad R, Srivastava AS, Singh AB. Cancer stem cells and colorectal cancer: an overview. Curr Top Med Chem. 2011; 11(13):1592-8.

54. Buczacki S, Davies RJ, Winton DJ. Stem cell quiescence and rectal carcinoma: an unexplored relationship and potential therapeutic target. $\mathrm{Br} J$ Cancer. 2011; 105(9):1253-9.

55. Rizk P, Barker N. Gut stem cells in tissue renewal and disease: methods, markers, and myths. Wiley Interdiscip Rev Syst Biol Med. 2012; 4(5):475-96.
56. Forster SJ, Talbot IC, Clayton DG, Critchley DR. Tumour basement membrane laminin in adenocarcinoma of rectum: an immunohistochemical study of biological and clinical significance. Int J Cancer.1986; 37(6):813-7.

57. Skovbjerg H, Anthonsen D, Lothe IM et al. Collagen mRNA levels changes during colorectal cancer carcinogenesis. BMC Cancer. 2009; 9: 136.

58. Shinto E, Tsuda H, Ueno $\mathrm{H}$ et al. Prognostic implication of laminin-5 gamma 2 chain expression in the invasive front of colorectal cancers, disclosed by area-specific four-point tissue microarrays. Lab Invest. 2005; 85(2):257-66.

59. Kirkland SC, Henderson K. Collagen IV synthesis is restricted to the enteroendocrine pathway during multilineage differentiation of human colorectal epithelial stem cells. J Cell Sci. 2001; 114(Pt 11):2055-64.

60. Kirkland SC. Type I collagen inhibits differentiation and promotes a stem cell-like phenotype in human colorectal carcinoma cells. Br J Cancer. 2009; 101(2):320-6.

61. Mauney J, Volloch V. Collagen I matrix contributes to determination of adult human stem cell lineage via differential, structural conformation-specific elicitation of cellular stress response. Matrix Biol. 2009; 28(5):251-62.

62. Schenke-Layland K, Angelis E, Rhodes KE et al. Collagen IV induces trophoectoderm differentiation of mouse embryonic stem cells. Stem Cells. 2007; 25(6):1529-38.

63. Lazaris ACh, Tzoumani AN, Thimara I et al. Immunohistochemical assessment of basement membrane components in colorectal cancer: prognostic implications. J Exp Clin Cancer Res. 2003; 22(4):599-606.

64. Valadi H, Ekstrom K, Bossios A et al. Exosomemediated transfer of mRNAs and microRNAs is a novel mechanism of genetic exchange between cells. Nat Cell Biol. 2007; 9: 654-59.

65. Clarke SL, Betts GJ, Plant A et al. CD4+CD25+FOXP3+ regulatory T cells suppress anti-tumor immune responses in patients with colorectal cancer. PLOS One. 2006; 1:e129.

66. Moinfar F, Man YG, Bratthauer GL et al. Genetic abnormalities in mammary ductal intraepithelial neoplasia-flat type ("clinging ductal carcinoma in situ"): a simulator of normal mammary epithelium. Cancer. 2000; 88(9):2072-81.

67. Malins DC, Gilman NK, Green VM. A cancer DNA phenotype in healthy prostates, conserved in tumors and adjacent normal cells, implies a relationship to carcinogenesis. Proc Natl Acad Sci USA. 2005; 102: 19093-6.

68. Pentheroudakis G, Briasoulis E, Pavlidis N. Cancer of unknown primary site: missing primary or missing biology? Oncologist. 2007; 12(4):418-25.

69. Hanauske AR, Clark GM, Von Hoff DD. Adenocarcinoma of unknown primary: retrospective analysis of chemosensitivity of 313 freshly explanted tumors in a tumor cloning system. Invest New Drugs.1995; 13(1):43-9.

70. Stoyianni A, Goussia A, Pentheroudakis G et al. Immunohistochemical study of the epithelial-mesenchymal transition phenotype in cancer of unknown primary: incidence, correlations and prognostic utility. Anticancer Res. 2012; 32(4):1273-81.

71. Alga AM, Rothwell PM. Effects of regular asprin on long-term cancer incidence and metastasis: a systematic comparison of evidence from observational studies versus randomised trials. Lancet Oncol. 2012; Epub ahead of print. 


\section{Figures}
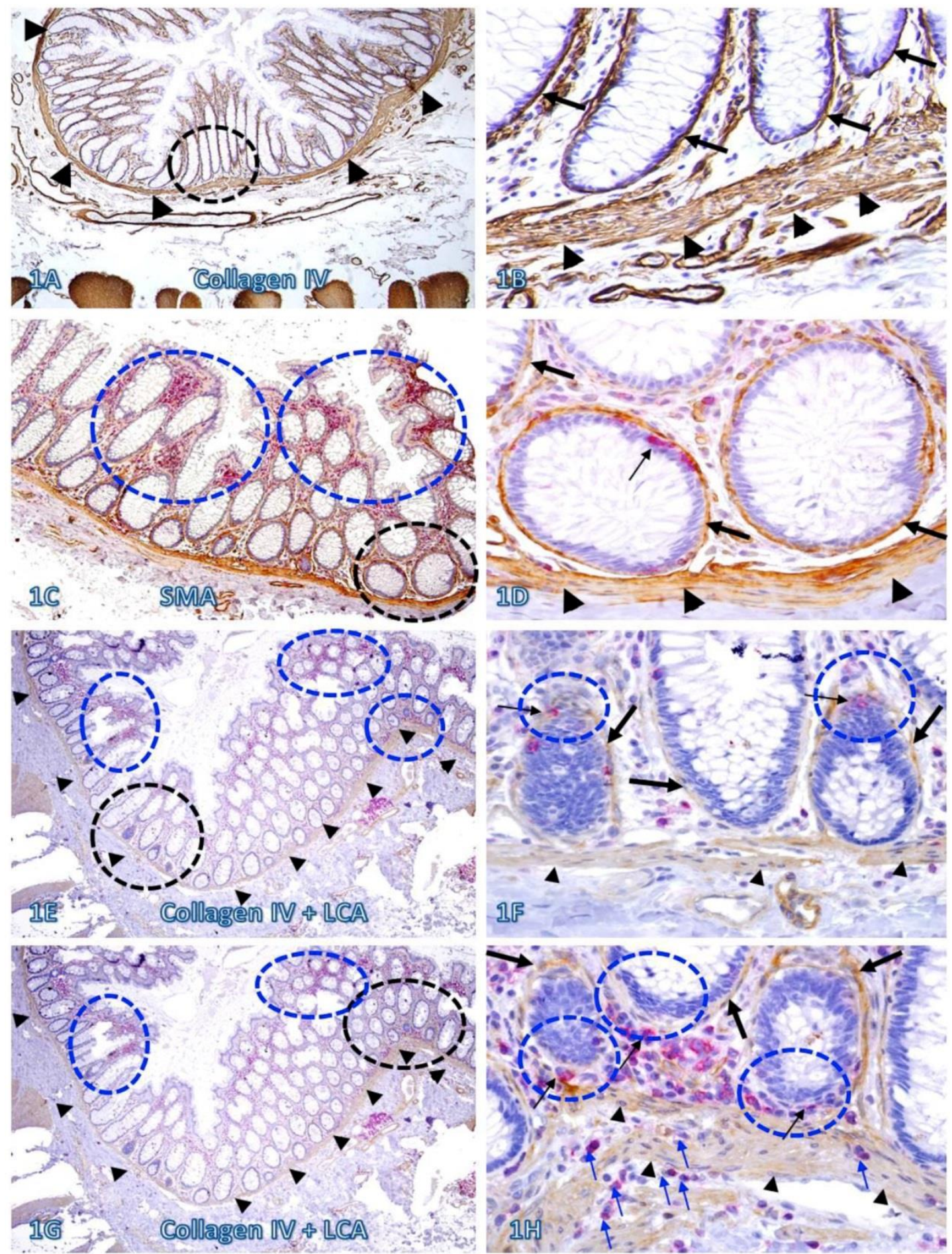

Fig I. The BM and MM status in normal tissues with and without infiltrating immune cells. Human normal colonic tissue sections were immunostained with different markers. Black circles identify low magnification views of the structures in $B, D, F$, and $H$, respectively. Blue circles identify epithelial structures with infiltrating immune cell aggregates in $E$ and $G$, and epithelial structures with focally disrupted $B M$ in $F$ and $H$. Thick arrows identify the BM. Thin arrows identify infiltrating immune cells. Arrowheads identify the MM. Note that both the BM and MM distant from infiltrating immune cells are densely packed and continuous. However, the BM and MM adjacent to infiltrating immune cells are generally attenuated or focally disrupted. Figures IE-H (which are a set of two adjacent sections) show two different patterns of focal BM disruptions: (a) disruptions facing towards the luminal direction, and (b) disruptions facing the MM. Note that the epithelial structures with focal BM disruptions facing the MM harbor budding and dissociated epithelial cells and the MM is loosely packed with "channel"-like spaces filled with a significant number of immune cells. In contrast, the BM in epithelial structures with focal BM disruption facing the luminal direction shows no distinct change. A, C, E, and G: 80X. B, D, F, and H. A higher (400X) of A, C, E, and G, respectively. 

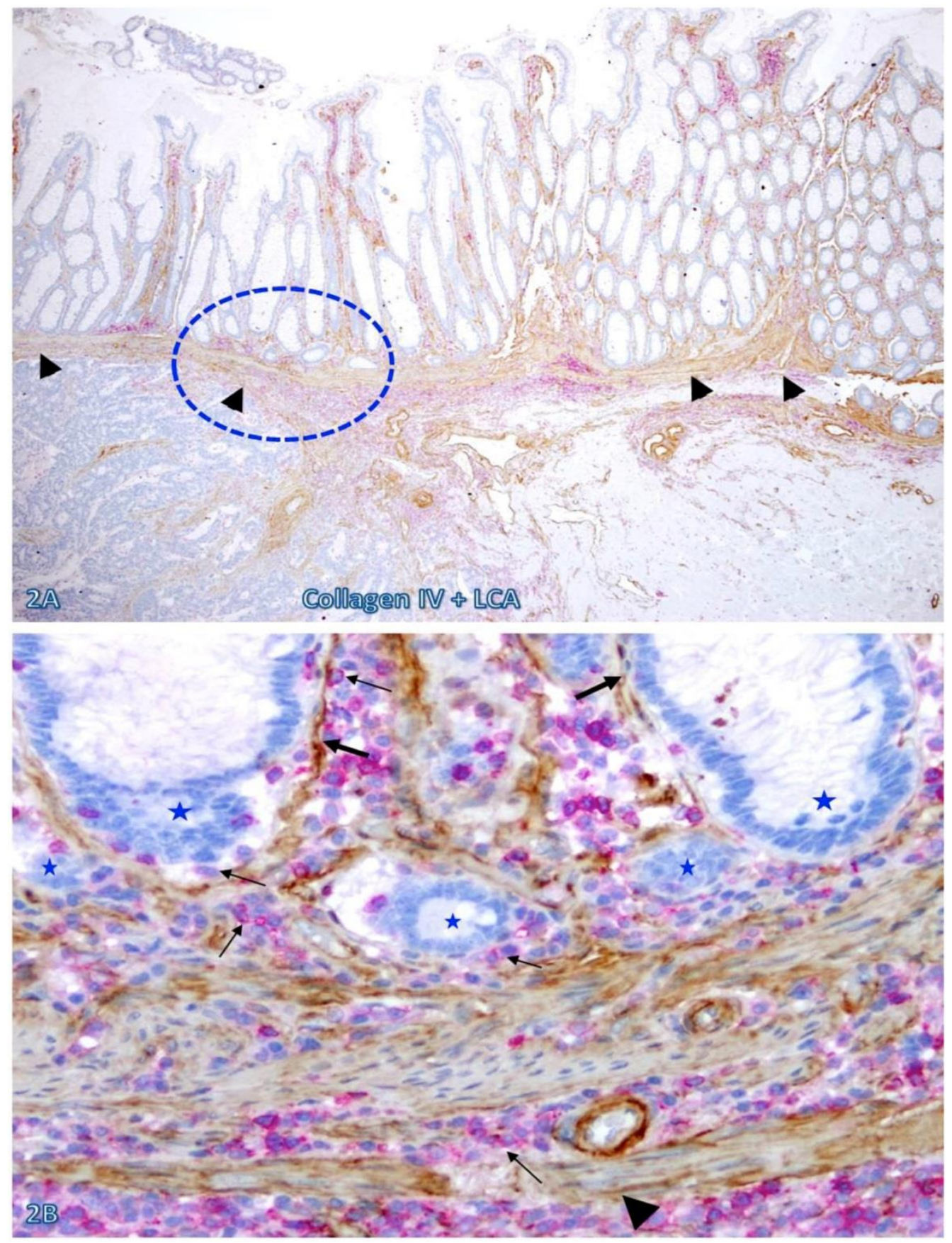

Fig 2. The BM and MM status in epithelial structures adjacent to invasive CRC. A CRC tissue section were double immunostained for collagen IV (brown) and LCA (red). A circle identifies the low magnification view of the structure in B. Thick and thin arrows identify the BM and infiltrating immune cells, respectively. Stars identify epithelial structures with no or focally disrupted BM. Arrowheads identify the MM. Note that the MM distant from infiltrating immune cell aggregates is well defined and continuous, while is substantially attenuated adjacent to the aggregates, Also note that the infiltrating immune cell aggregate harbor several epithelial structures with no or focally disrupted BM. A: 80X. B: 400X. 

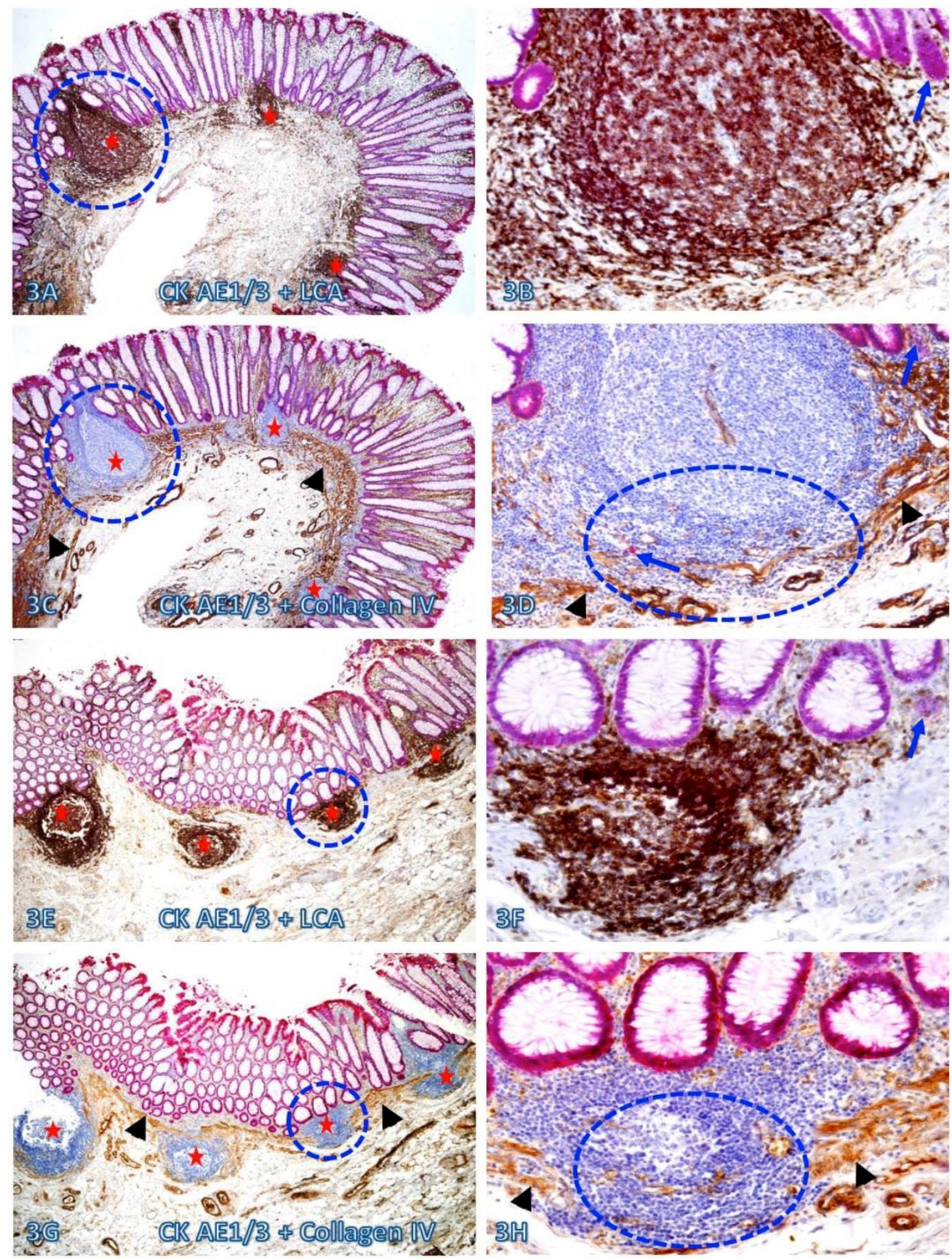

Fig 3. Focal fragmentations in the MM adjacent to lymphoid follicles. Two sets of two adjacent ( $A-D$ and $E-H)$ normal colonic tissue sections at a distance from CRC from two different cases were double immunostained for CK AEI/3 (red) plus LCA or collage IV (brown). Smaller circles identify the low magnification views of the structures in $\mathrm{B}, \mathrm{D}, \mathrm{F}$, and $\mathrm{H}$. Larger circles in $\mathrm{F}$ and $\mathrm{H}$ identify gaps in the MM. Stars identify lymphoid follicles. Arrowheads identify the MM. Arrows identify dissociated epithelial cells or cell clusters without the surrounding BM. Note that the MMs adjacent to all 7-lymphoid follicles are focally disrupted. A, C, E, and G: 80X. B, D, F, and H: a higher magnification (400X) of A, C, E, and G, respectively. 


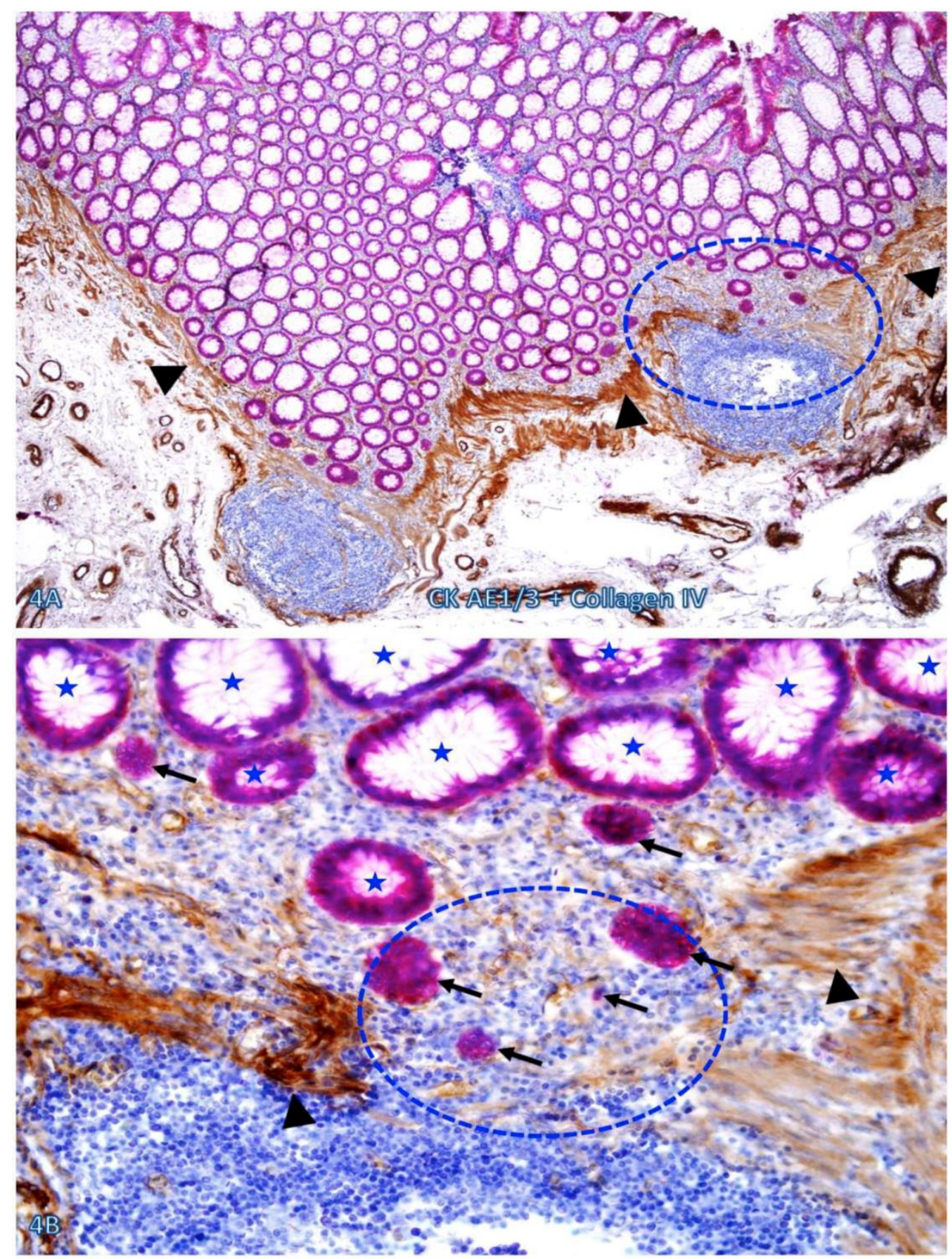

Figure 4. Dissociated cells or cell clusters overlying disruptions in MM near lymphoid follicle. A normal colon tissue section from a node-positive case was double immunostained for CK AEI/3 (red) and collagen IV (brown). The circle identifies the low magnification view of the structure in $B$, and the circle in B identifies the gap in the MM. Arrows identify solid epithelial cell clusters without a distinct BM overlying the focal disruption in the MM. Arrowheads identify the MM. Stars identify normal epithelial structures with a distinct lume (stars). Note that a vast majority of the epithelial structures distant from lymphoid follicles are uniform in size with a distinct lumen, while isolated solid cell clusters without a lumen and the surrounding BM are exclusively seen overlying the focal disruption in the MM. A: 80X. B: 400X 

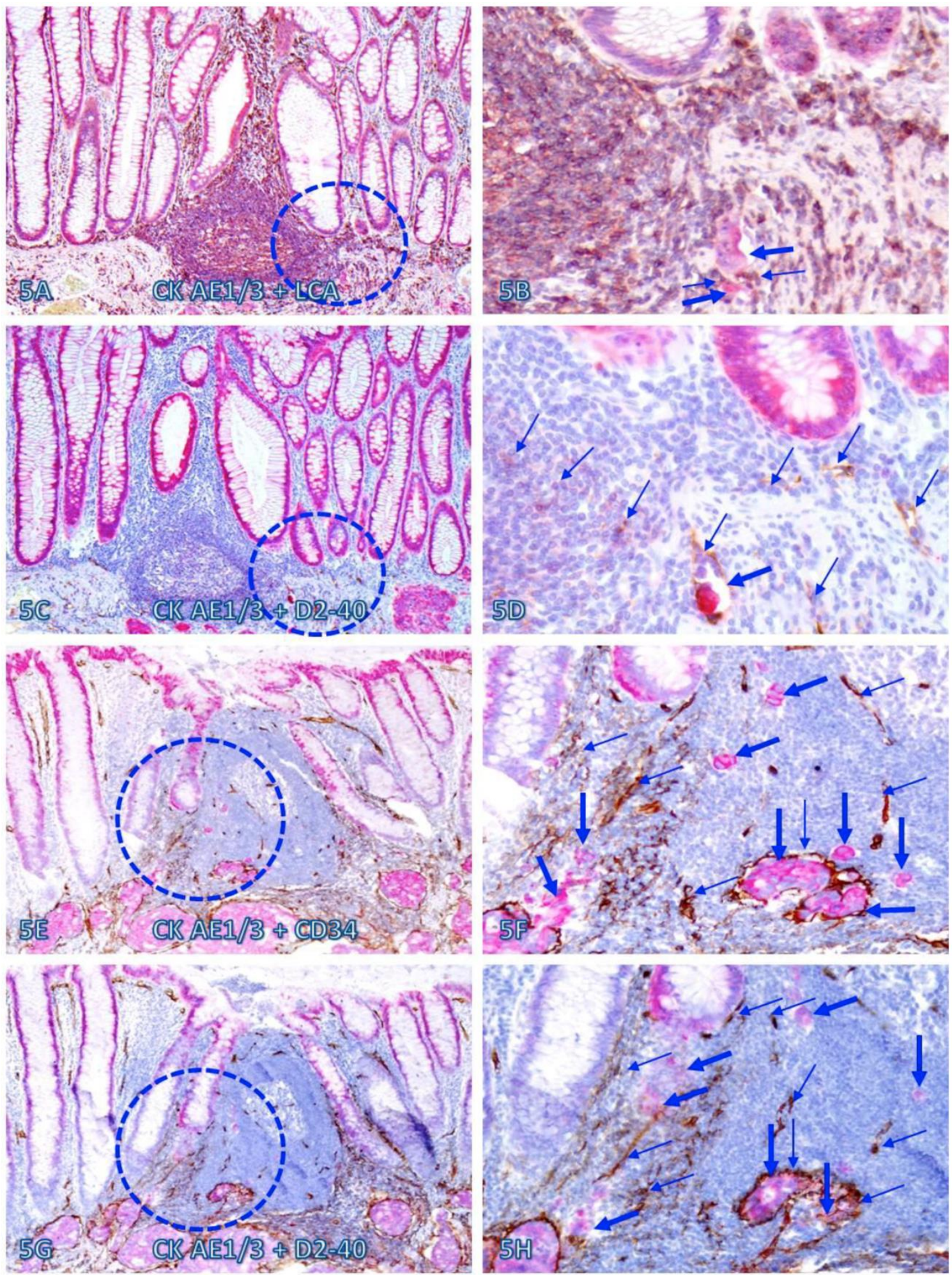

Fig 5. Dissociated epithelial cells within lymphoid follicles with increased vascular density. Two sets of two adjacent CRC tissue sections (A-D and E-H) from two node-positive cases were double Immunostained for CK AEI/3 (red) plus LCA or D2-40 (brown) or for CK-19 (red) plus CD34 or D2-40 (brown). Circles identify the low magnification views of the structures in B, D, F, and H. Thick and thin arrows identify dissociated epithelial cells and associated lymphocytes $(B)$ or lymphatic ducts $(D, F$, and $H)$, respectively. Note that the vascular density in each of the follicles is substantially increased and some disseminated cells are located within these follicles. A, C, E, and G: I00X. B, D, F, and H: a higher magnification (300X) of A, C, E, and G, respectively. 

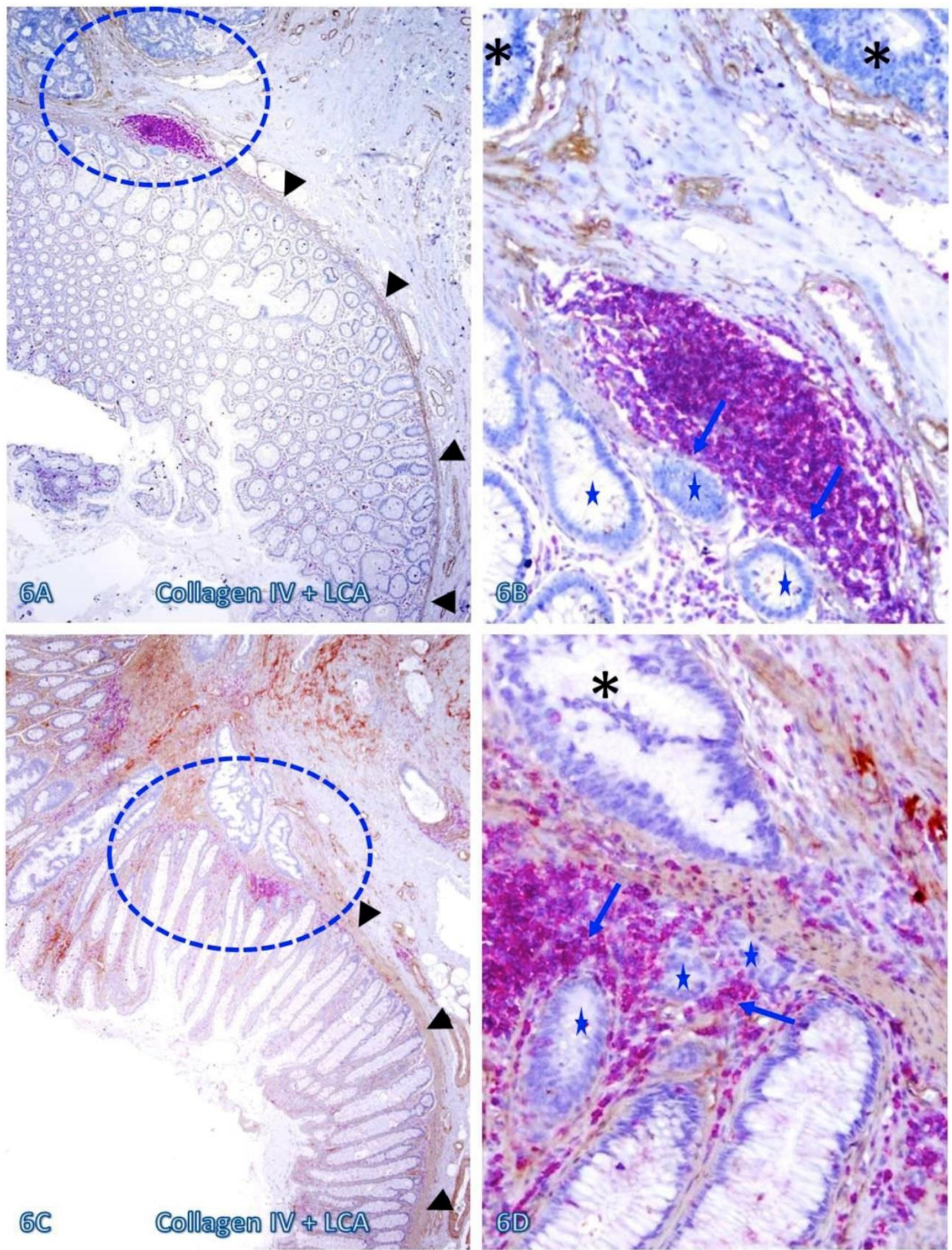

Fig 6. Invasive CRC adjacent to lymphoid follicles and aggregates. CRC tissue sections from two cases were double immunostained for collagen IV (brown) and LCA(red). Circles identify the low magnification views of the structures in B and D. Asterisks identify the invasive CRC. Stars identify epithelial structures within or adjacent to lymphocyte aggregates with no distinct BM. Arrows identify infiltrating immune cells. Note the MM distant from the lymphocyte aggregates is well defined and continuous, while it is focally disrupted at and near these aggregates. Also note that multiple epithelial cell clusters adjacent to these lymphocytes are morphologically similar to invasive cancer. A and C: $80 X$. B and D: a higher magnification (400X) of A and C, respectively. 

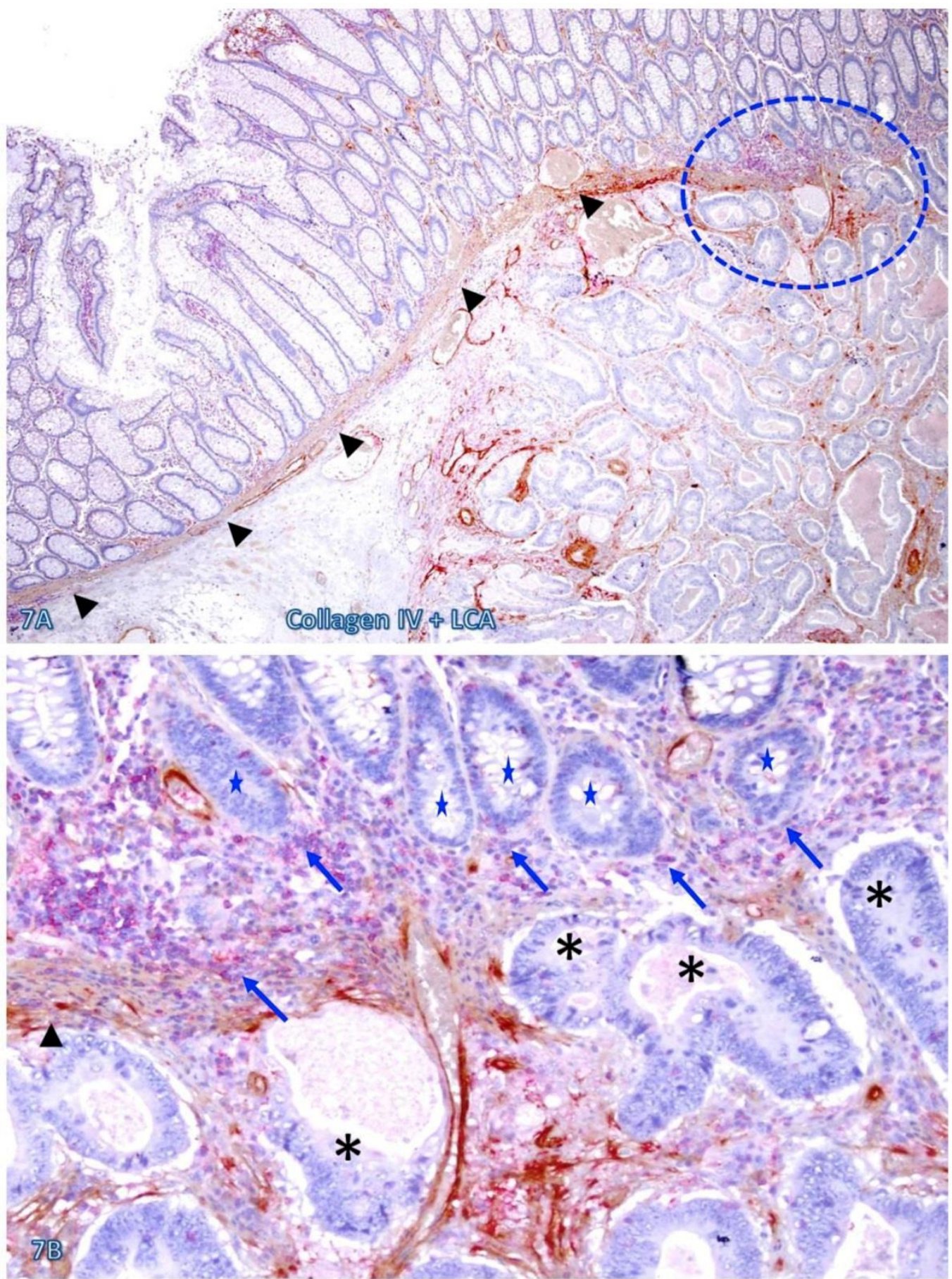

Fig 7. CRC invasion within lymphoid aggregates. A CRC tissue section was double immunostained for collagen IV (brown) and LCA (red). The circle identifies the low magnification views of the structures in B. Asterisks identify the invasive CRC. Stars identify epithelial structures within or adjacent to lymphocyte aggregates with no distinct BM. Arrows identify infiltrating immune cells. Note the MM distant from the lymphocyte aggregates is well defined and continuous, while it is focally disrupted at and near lymphocyte aggregates. Also note that multiple epithelial cell clusters adjacent to these lymphocytes are morphologically similar to invasive cancer. A: 80X. B: a higher magnification (400X) of A. 


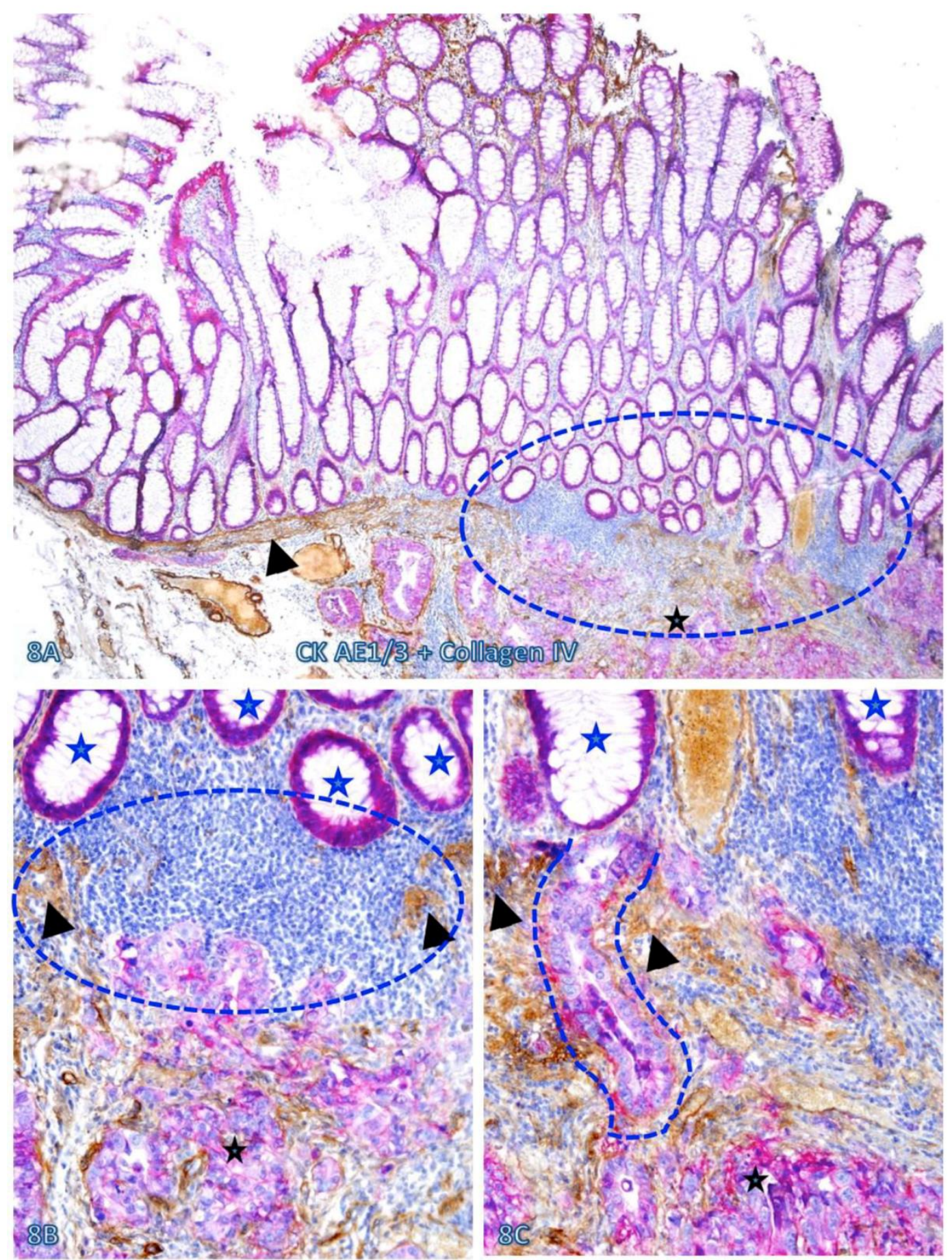

Fig 8. Pre-invasive-invasive junction adjacent to large lymphocyte aggregates. $A C R C$ tissue section was double immunostained for $C K A E I / 3$ (red) and collagen IV (brown). The circle in A identifies the low magnification views of the structures in B and C, and the circle in B identifies a gap in the MM (arrowheads). Black and blue stars identify the invasive lesion and normal epithelial structures, respectively. Curve lines identify a tongue-like epithelial cell projection invading the submucosa through focally disrupted MM. Note that this cell projection appears to be directly budding from a normal epithelial structure. A: 80X. B: A higher (200X) of A. 


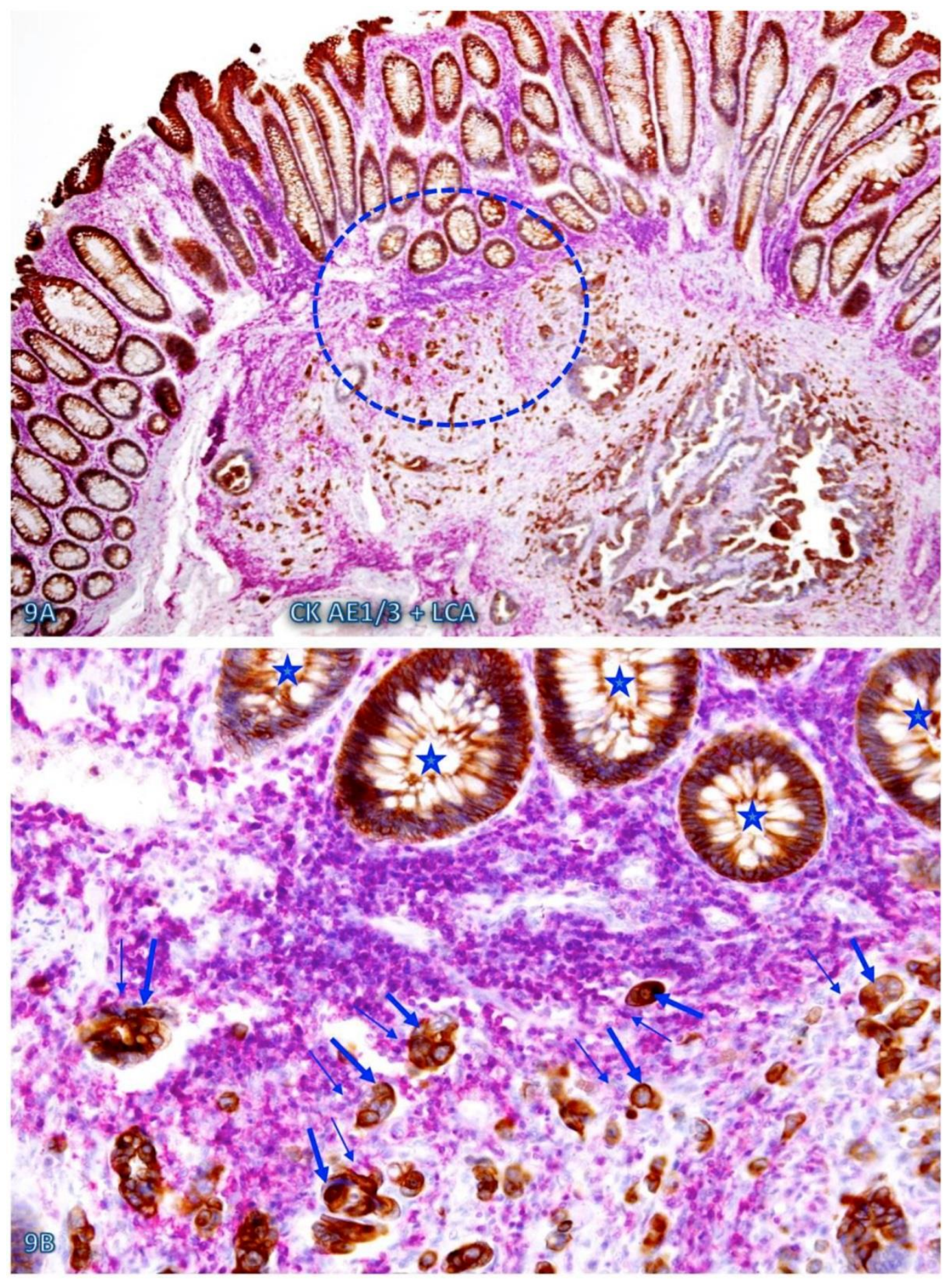

Fig 9. Cell budding from normal epithelial structures at pre-invasive-invasive junction. A CRC tissue section was double immunostained for CK AEI/3 (red) and LCA (brown). The circle in A identifies the low magnification views of the structures in B. Stars identify normal appearing epithelial structures at the pre-invasive-invasive junction. Thick and thin arrows identify dissociated epithelial cells and their associated lymphocytes, respectively. Note that the entire section harbor no morphologically distinct intra-mucosal carcinoma, while many dissociated epithelial cells or cell clusters are seen at the pre-invasive and invasive junction. These dissociated epithelial cells appear to be directly budding from the normal epithelial structures (stars). Also note that the pre-invasive-invasive junction and dissociated epithelial cells are exclusively seen adjacent to the lymphocyte aggregates. A: 80X. B: A higher (200X) of $A$. 


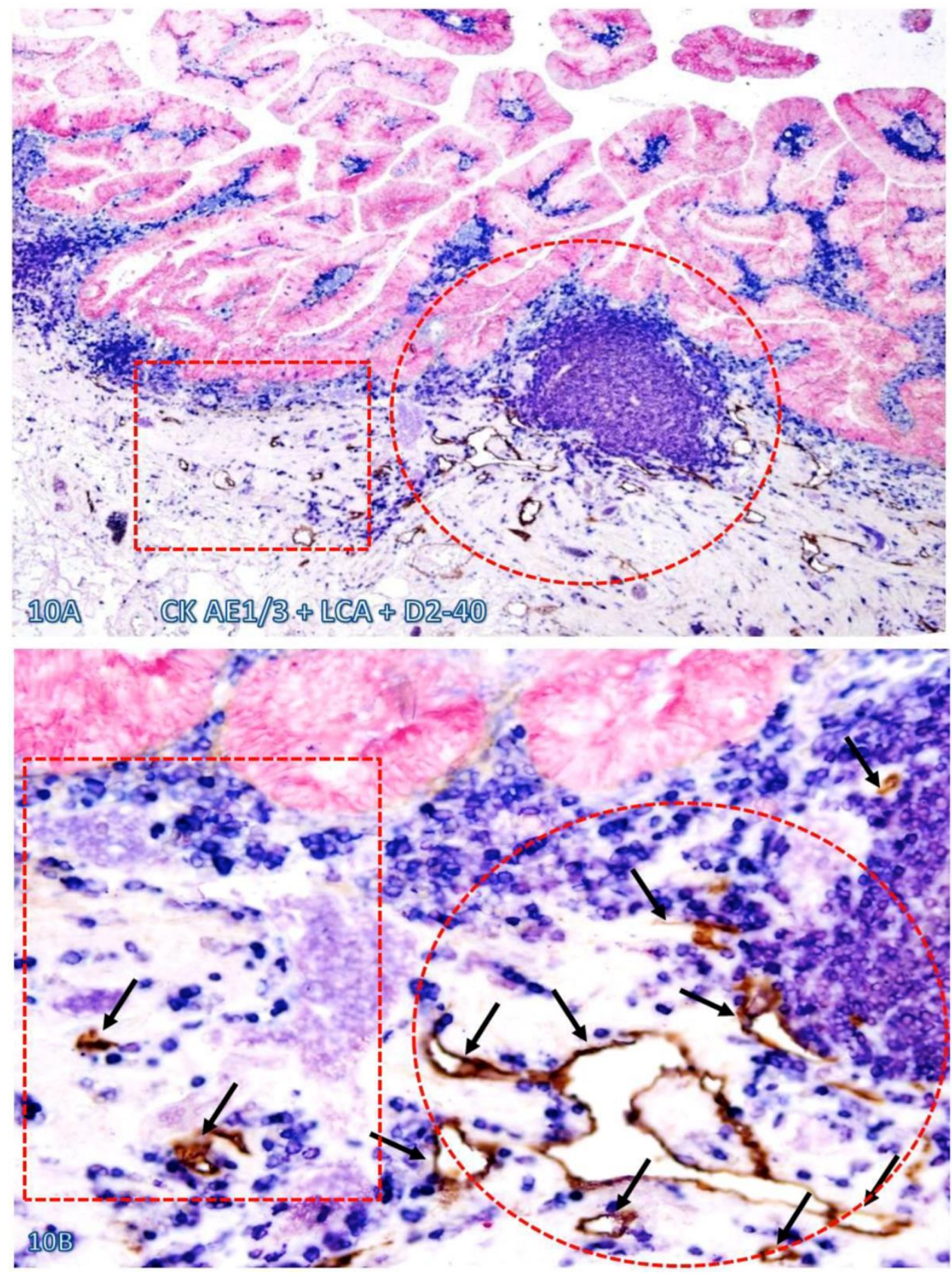

Fig 10. Significantly increased lymphatic duct density near lymphocyte aggregates. A normal colonic tissue sections distant from CRC was triple immunostained for CK AEI/3 (red), LCA (blue) and D2-40 (brown). Circles and squares identify tissues adjacent to and distant from an infiltrating immune cell aggregate, respectively. Note that substantially more and larger lymphatic ducts (arrows) are seen in the tissue adjacent to than distant from, the infiltrating immune cell aggregate. A: $80 \mathrm{X}$. B: a higher (400X) magnification of $\mathrm{A}$. 


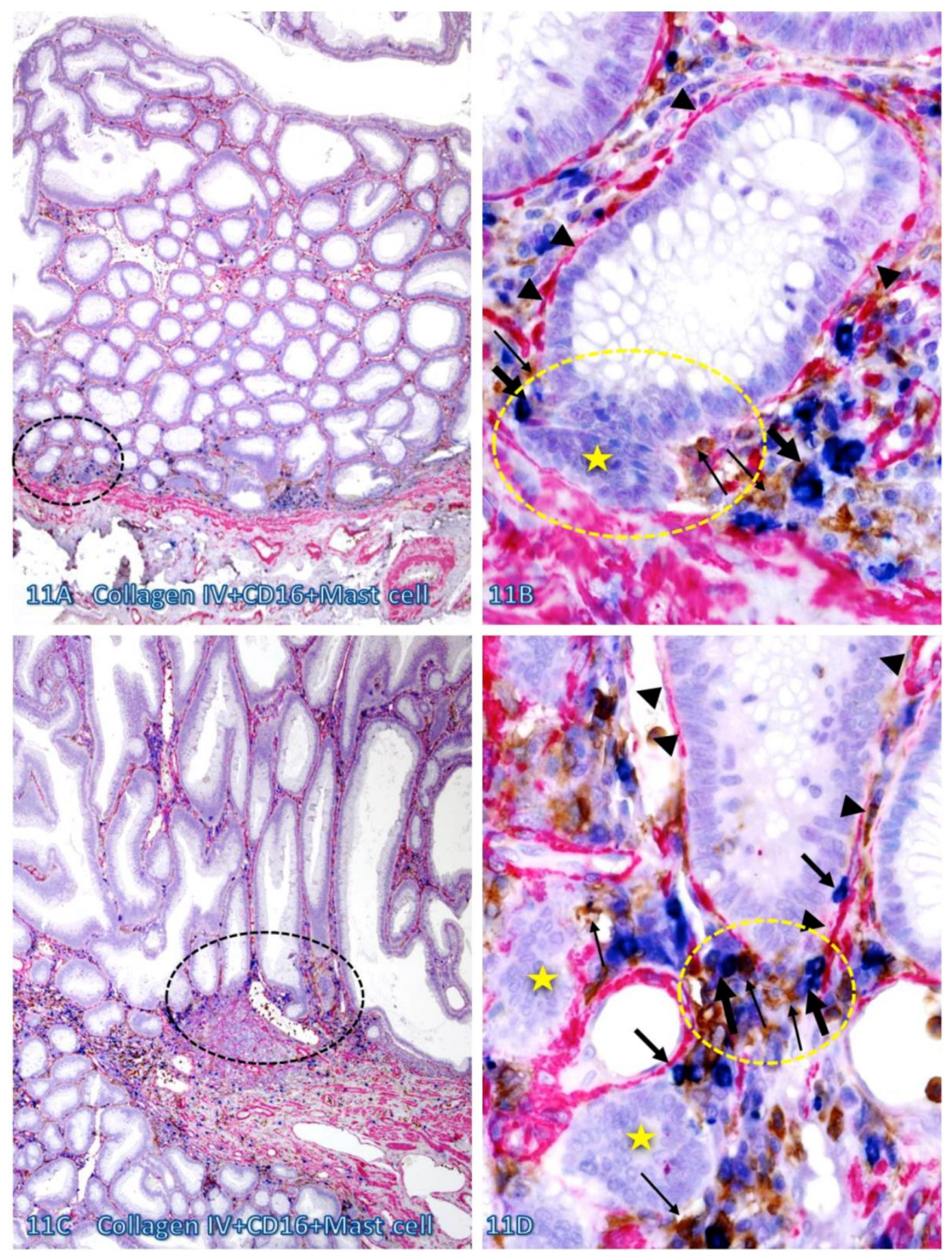

Fig I I. Direct physical contact between the BM and infiltrating immune cells. Two normal colonic tissue sections distant from CRC were triple immunostained for collagen IV (red), CDI6 (NK cells; brown), and Mast cells (blue). Black circles identify the low magnification view of the structures in B and D. Yellow circles identify focal disruptions in the epithelial capsules (arrowheads). Thin and thick arrows identify NK and Mast cells, respectively. Stars identify dissociated cell clusters without a distinct surrounding BM. Note that infiltrating NK and Mast cells are preferentially located at or near the site of focal disruptions in the epithelial capsule. The BM without associated infiltrating immune cells is generally continuous and well-defined. A and C: I00X. B and D: a higher (500X) magnification of $A$ and $C$, respectively. 

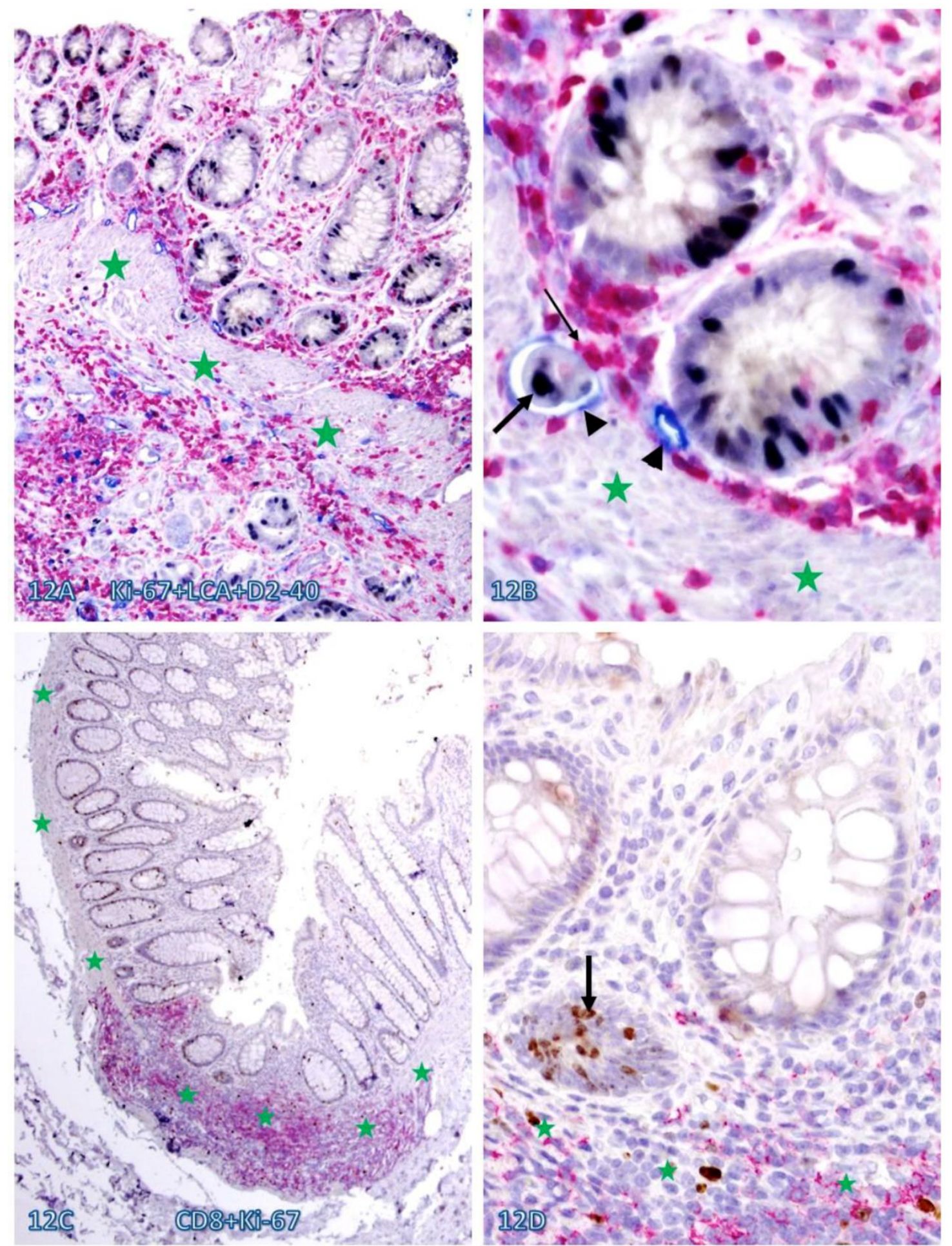

Fig 12. Elevated cell proliferation in cell cluster adjacent to infiltrating immune cells. A CRC (A-B) section was triple immunostained for LCA (red), Ki-67 (black), and D2-40 (blue) and a normal (C-D) colonic section was double immunostained for CD8 (red) and Ki-67 (brown). Circles identify the low magnification views of the structures in B and D. Stars identify the MM. Arrowheads identify lymphatic ducts. Thin and thick arrows identify infiltrating immune cells and proliferating epithelial cells, respectively. Note that dissociated epithelial cells within the lymphatic duct (A-B) or the stroma (C-D) had a higher proliferation identix than their adjacent counterparts. A and C: I00X. B and D: a higher (400X) magnification of $A$ and $C$, respectively. 

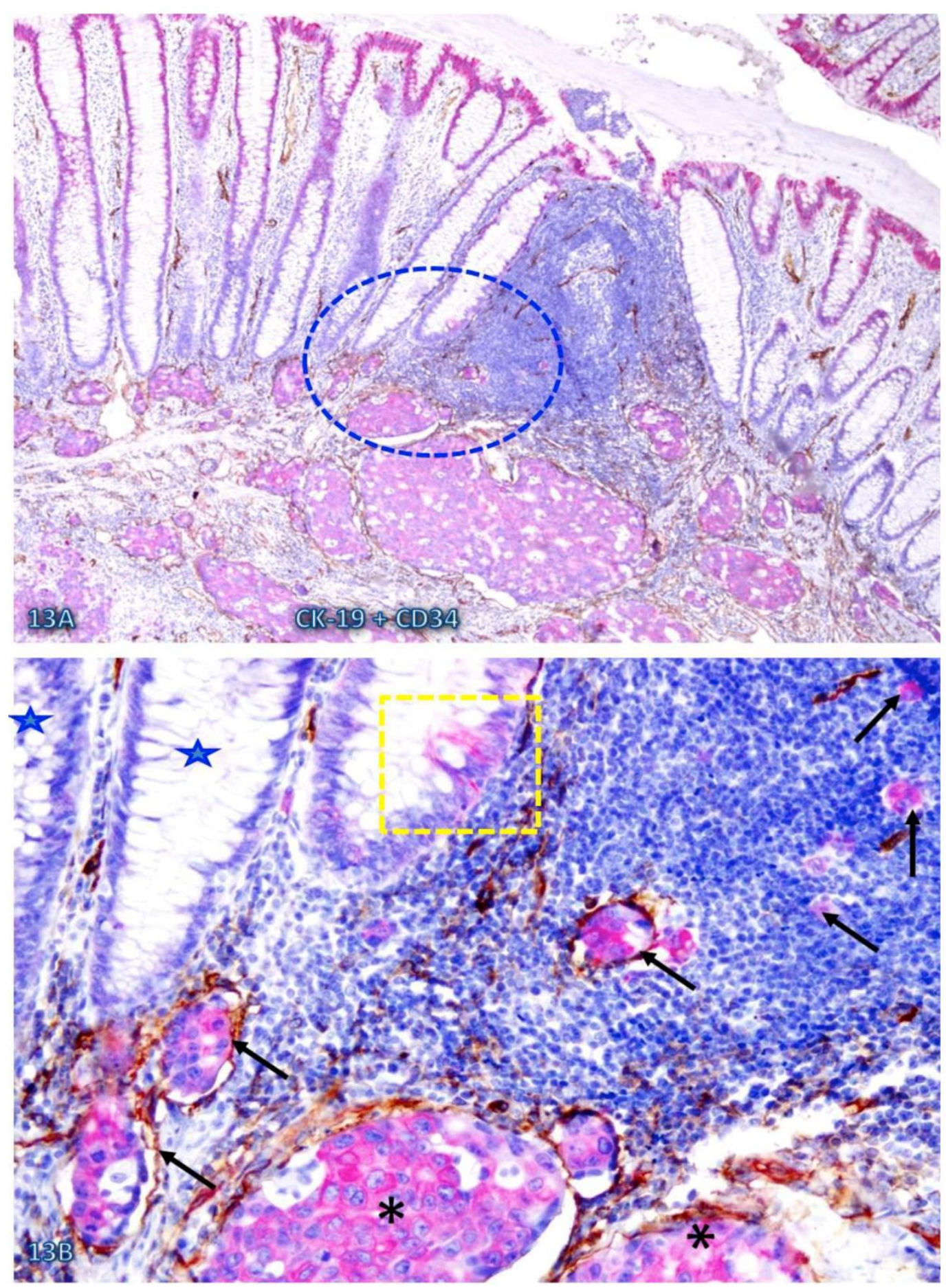

Fig 13. Simiilarity among budding CK-19 positve, invasive, and disseminated cells. A CRC tissue section was double immunostained for CK-19 (red) and CD34 (brown). A circle identifies the low magnification view of the structures in B. A square identifies a cluster of CK-19-positive cells located within an overall CK-19-negative epithelial structure. Stars identify normal CK-19-negative epithelial structures. Asterisks identify invasive cancer. Arrows identify dissociated or disseminated CK-19-posive cell clusters. Note that these budding cells are immunohistochemically and morphologically similar to invasive and disseminated cells within the vascular structure. A: 80X. B: a higher (300X) magnification of A. 

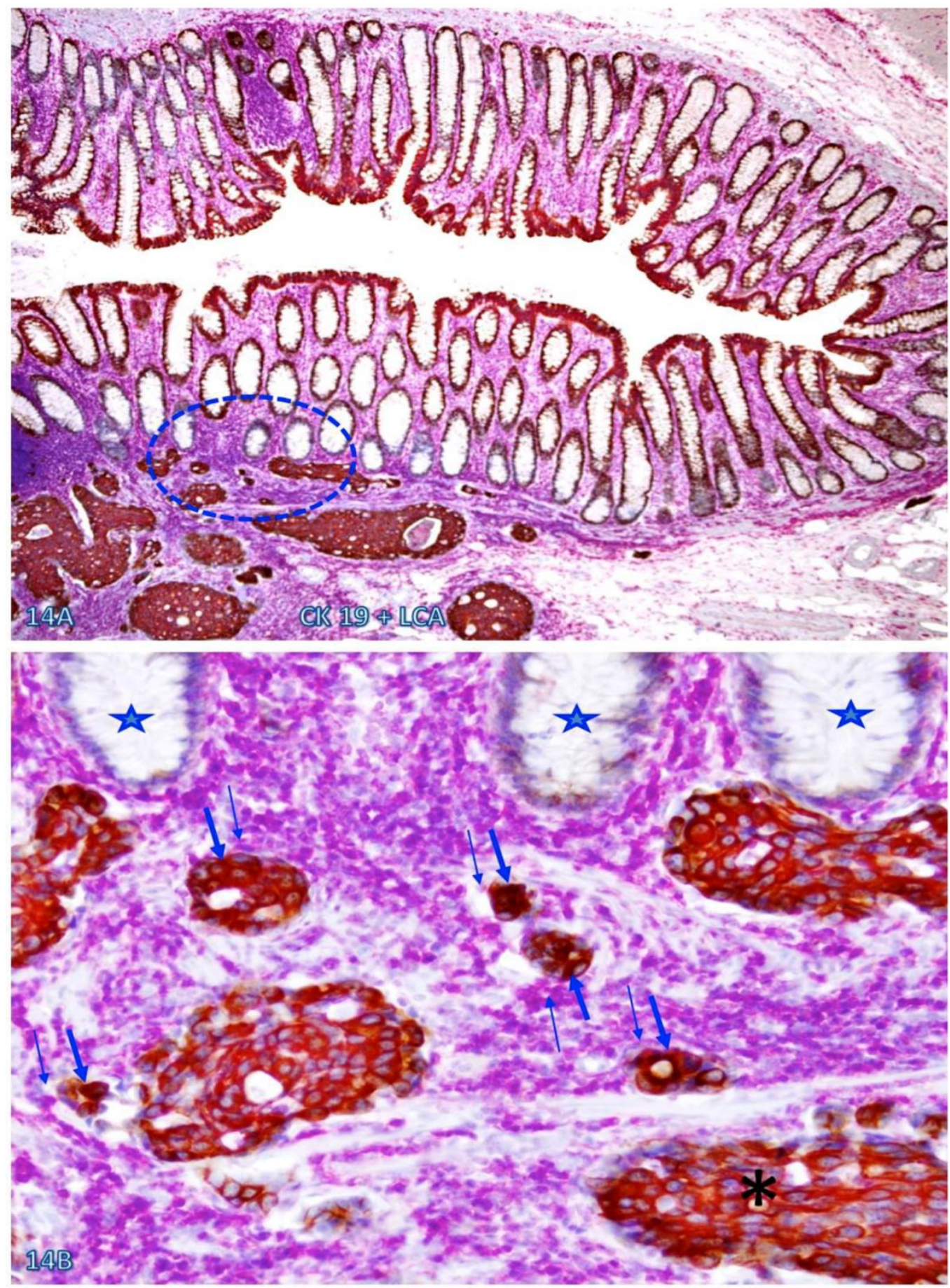

Fig 14. CK-19-positive cell cluster budding form CK-19-negative epithelial structures. A CRC tissue section was double immunostained for CK-19 (brown) and LCA (pink). A circle identifies the low magnification view of the structures in B. Stars identify normal CK-19-negative epithelial structures. Thick and thin arrows identify CK-19-posive cell clusters and physically associated immune cells, respectively. Note that these budding cells are either surrounded by or physically conjoined with infiltrating immune cells. Also note that these budding cells are immunohistochemically and morphologically similar to invasive cancer cells (asterisk). A: 80X. B: a higher (300X) magnification of A. 